\title{
Identification of pathways directly regulated by SHORT VEGETATIVE PHASE during vegetative and reproductive development in Arabidopsis
}

\author{
Veronica Gregis ${ }^{1 \dagger}$, Fernando Andrés ${ }^{2 \dagger}$, Alice Sessa ${ }^{1 \dagger}$, Rosalinda F Guerra ${ }^{1}$, Sara Simonini ${ }^{1}$, Julieta L Mateos ${ }^{2}$, \\ Stefano Torti ${ }^{2}$, Federico Zambelli', Gian Marco Prazzoli ${ }^{1}$, Katrine N Bjerkan ${ }^{3}$, Paul E Grini ${ }^{3}$, Giulio Pavesi ${ }^{1}$, \\ Lucia Colombo ${ }^{1,4}$, George Coupland ${ }^{2}$ and Martin M Kater ${ }^{1 *}$
}

\begin{abstract}
Background: MADS-domain transcription factors play important roles during plant development. The Arabidopsis MADS-box gene SHORT VEGETATIVE PHASE (SVP) is a key regulator of two developmental phases. It functions as a repressor of the floral transition during the vegetative phase and later it contributes to the specification of floral meristems. How these distinct activities are conferred by a single transcription factor is unclear, but interactions with other MADS domain proteins which specify binding to different genomic regions is likely one mechanism.

Results: To compare the genome-wide DNA binding profile of SVP during vegetative and reproductive development we performed ChIP-seq analyses. These ChIP-seq data were combined with tiling array expression analysis, induction experiments and qRT-PCR to identify biologically relevant binding sites. In addition, we compared genome-wide target genes of SVP with those published for the MADS domain transcription factors FLC and AP1, which interact with SVP during the vegetative and reproductive phases, respectively.

Conclusions: Our analyses resulted in the identification of pathways that are regulated by SVP including those controlling meristem development during vegetative growth and flower development whereas floral transition pathways and hormonal signaling were regulated predominantly during the vegetative phase. Thus, SVP regulates many developmental pathways, some of which are common to both of its developmental roles whereas others are specific to only one of them.
\end{abstract}

Keywords: MADS-box, gene regulation, transcription factors, post transcriptional regulation, ChIP-seq, floral transition, floral development, Arabidopsis thaliana

\section{Background}

In plants organs are formed post-embryonically from populations of undifferentiated cells called meristems. In these meristems, stem cell activity is kept at the central zone whereas at the peripheral part of the meristem primordia arise in which cells differentiate into organs. In flowering plants like Arabidopsis thaliana during the vegetative phase the primordia that derive from the shoot apical meristem (SAM) develop into leaves $[1,2]$.

\footnotetext{
* Correspondence: martin.kater@unimi.it

+ Contributed equally

'Department of Bioscience, Università degli Studi di Milano, Via Celoria 26, 20133 Milan, Italy

Full list of author information is available at the end of the article
}

The change to the subsequent generative phase is called floral transition, which is regulated by multiple flowering pathways that are controlled by environmental and endogenous cues. During the floral transition, the SAM undergoes a change in fate and becomes an inflorescence meristem (IM). The Arabidopsis IM is an indeterminate meristem and develops multiple determinate floral meristems (FMs) in a spiral manner, which in turn produce a precise number of floral organs arranged in a whorled pattern $[1,3,4]$. The reprogramming of meristems is regulated by a complex gene regulatory network in which transcription factors represent important key players.

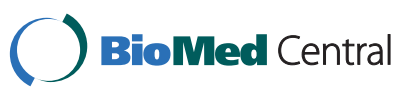

() 2013 Gregis et al.; licensee BioMed Central Ltd. This is an open access article distributed under the terms of the Creative Commons Attribution License (http://creativecommons.org/licenses/by/2.0), which permits unrestricted use, distribution, and reproduction in any medium, provided the original work is properly cited. 
In Arabidopsis the photoperiod, thermosensory, and vernalization/autonomous pathways that respond to environmental signals, and the aging and gibberellic acid pathways that respond to the developmental and physiological state of the plant regulate the floral transition [5]. Many transcription factors encoding genes have been shown to be involved in the regulation of these pathways including those belonging to the MADS-box gene family $[6,7]$. One of these MADS-box genes controlling flowering time is SHORT VEGETATIVE PHASE (SVP) [8].

MADS-domain transcription factors have been identified in all eukaryotic kingdoms and in Arabidopsis thaliana they are involved in most important developmental processes [9-12]. MADS-domain factors activate or repress transcription by direct binding to short sequences called CArG-boxes that correspond to a 10 nucleotide sequence $\mathrm{CC}(\mathrm{A} / \mathrm{T})_{6} \mathrm{GG}$ present in the regulatory sequences of target genes. However, this motif can be quite variable allowing some mismatches $[10,13]$. Moreover MADS-domain proteins form homo and/or heterodimers and are also suggested to form tetrameric MADS-domain complexes [14]. The variety of interactions that many MADS-domain factors can make suggests that they may regulate different subsets of genes during different phases of development and might reflect an enormous regulatory potential [15]. Furthermore, their association with others co-factors probably also influences the affinity and specificity of the complex for specific target sequences $[16,17]$.

During the vegetative phase $S V P$ acts as a repressor of flowering since the $s v p$ mutant flowers very early [8]. SVP mediates flowering responses by perceiving signals from different endogenous and environmental flowering pathways such as the thermosensory, autonomous, and GA pathways $[6,18]$. SVP regulates the expression of three floral pathways integrator genes (FPI) that are FLOWERING LOCUS T (FT), TWIN SISTER OF FT (TSF), and SUPPRESSOR OF OVEREXPRESSION OF CONSTANS 1 (SOC1) which all promote flowering $[18,19]$. To maintain plants in the vegetative phase, SVP represses the expression of $F T$ and TSF in the phloem and SOC1 in the SAM by directly binding to CArG boxes in FT and $S O C 1$ $[6,18,19]$. During the vegetative phase, SVP interacts with another central repressor of flowering time that is FLOWERING LOCUS C (FLC) and their function is mutually dependent. In fact it has recently been demonstrated that the SVP-FLC dimer acts to directly repress $F T$ in the leaves and SOC1 in the SAM [18]. During the floral transition, SVP expression gradually decreases until the SVP protein completely disappears from the IM [20]. In plants competent to flower, inputs deriving from the flowering pathways converge to repress SVP and FLC expression $[18,19]$. During the vegetative phase SVP plays an opposite role to its phylogenetically closest related MADS-box gene AGAMOUS LIKE 24 (AGL24), which is a central promoter of flowering [21,22]. Both SVP and AGL24 directly regulate $S O C 1$ by binding its promoter on the same binding sites but they have an opposite effect on SOC1 expression [23].

Interestingly, after the floral transition both $S V P$ and AGL24 are co-expressed in the floral meristem during stage 1 and 2 of flower development [24]. Analysis of the svp agl 24 double mutant, especially at higher temperatures, and the svp ap1 agl24 triple mutants showed that AGL24 and SVP play redundant roles during these early stages of flower development $[20,24,25]$. Combining the svp agl24 double mutant with a weak ap1 allele showed that AGL24 and SVP together with AP1 repress floral homeotic genes that control petal, stamen and carpel identity [25]. Protein interaction and genetic studies revealed that SVP and AGL24 are able to form dimers with AP1 and that this dimer is able to recruit the LEUNIG-SEUSS co-repressor complex $[15,25]$. Combining the svp agl24 double mutant with a strong ap 1 allele showed that they are also controlling floral meristem identity since this triple mutant forms on the flanks of the IM new IMs instead of FMs resulting in a cauliflower like curd just as observed in the ap1 cauliflower (cal) double mutant $[24,26]$. Recently Simonini et al. [17] have shown that the corepressor complex composed of LUG, SEU, and SVP is also able to repress the ovule identity gene SEEDSTICK (STK) in a complex together with BASIC PENTACYSTEINE transcription factors.

SVP is a key factor for Arabidopsis development and acts both during vegetative and reproductive phases where it plays different roles probably by interacting with different partners to regulate specific sets of target genes. Even though $S V P$ is a gene of interest since its first characterization [8], still little is known about the mode of action and the network of genes controlled by this MADS-domain transcription factor. A powerful tool to study in vivo the genome-wide DNA-binding patterns of transcription factors is the ChIP-seq technology that consists in ultra-high throughput Solexa (Illumina) sequencing of DNA samples obtained by chromatin immunoprecipitation (ChIP). This technique has been used for a few years to identify direct target genes. At first for human transcription factors like NRSF, STAT1, PPAR $\gamma$, and FOXA2 [27-30] and recently this technology has been reported for the identification in Arabidopsis of genome wide targets of different MADSdomain proteins such as, SEPALLATA3 (SEP3), AP1, FLC, and SOC1 [13,31-33] and another important transcriptional regulator such as AP2 [34]. Moreover genome wide binding site analysis is also possible using the ChIP on chip method, as was done for AGAMOUS LIKE 15 (AGL15), LEAFY (LFY), SVP, and SOC1 [35-37].

Here we report the use of the ChIP-seq approach to identify genome wide binding sites for SVP, during two distinct developmental phases: the vegetative and reproductive 
phase. This study allowed us to identify new pathways that are regulated by SVP in vegetative and reproductive tissues and to investigate genome-wide interaction dynamics of a transcription factor during different phases of development.

\section{Results}

\section{Genome-wide mapping of SVP binding sites during} vegetative and reproductive development

For genome-wide identification of the in-vivo binding sites of the SVP MADS-box transcription factor ChIP was performed followed by single end-read sequencing with the Solexa/Illumina GA platform. For the ChIP experiments Arabidopsis svp mutant plants expressing epitope tagged SVP were used [20]. The full genomic region of $S V P$ including $3 \mathrm{~kb}$ upstream of the start codon was cloned as a C-terminal fusion with GREEN FLUORESCENT PROTEIN (GFP) [38]. Since SVP plays important roles during two distinct non-overlapping phases of development, namely the floral transition [8] and the early stages (stages 1 and 2) of flower development $[20,24,25,39]$, studying the genome-wide binding sites of SVP provides an opportunity to compare the pathways directly regulated by SVP during these two developmental phases. Therefore vegetative phase material was harvested from 2-week-old seedlings grown under short-day conditions, whereas reproductive phase inflorescences with developing flowers of stage 1 to 11 [40] were harvested to analyze its targets during flower development.

Several independent ChIP experiments were performed. As control the same tissues were harvested from wild-type plants that did not express SVP-GFP. ChIP experiments that showed relatively high enrichment for known SVP binding regions ( $F T$ for the vegetative tissues and $A G$ for reproductive tissues) were used to select samples for sequencing (see Additional data file 1, Figure S1) $[6,20]$.

\section{Distribution of SVP binding sites across the genome and within genes}

For both vegetative and reproductive tissues as well as for the control, two independent ChIP reactions were sequenced. As in similar experiments $[13,31]$, sequence reads obtained from duplicate experiments for each of the three samples were pooled. Only reads mapping to a unique position on the genome were considered for further analysis. This resulted in about 3 million uniquely mapped reads for the two experiments using inflorescence material, 5 million for experiments performed using vegetative material, and 6 million for control experiments (Additional data file 1, Table S1).

The regions enriched for binding sites were then identified with a strategy broadly similar to the one previously employed for SEP3 and AP1 [13,31], and implemented in the CSAR tool [41]. At a Bonferroni-corrected $P$ value of 0.01 this resulted in about 13,000 regions in inflorescence tissues and 25,000 in seedlings, reduced to about 8,000 and 15,000, respectively, at threshold 10-4, and about 1,300 in both experiments at threshold 10-5 (see material and methods and Additional data file 2, Table S2). The overall distribution of SVP-binding sites across the genome in both tissues does not change significantly, and shows that $40 \%$ of the sites are located within the $3 \mathrm{~Kb}$ upstream of the gene, $27 \%$ in the transcribed region, whereas $4 \%$ are inside the $1 \mathrm{~Kb}$ downstream regions (Figure 1a). Regions falling within the transcribed regions tend to be located towards the 3' UTR/transcription termination (Figure 1b). A similar observation was made on the genome-wide distribution of SEP3 MADS-box protein binding sites [13]; moreover in Kaufmann et al. [31] they found that AP1 is able to bind the 3' region of TERMINAL FLOWER 1 (TFL1) which is an important shoot identity gene [42]. TFL1 3' region is indeed required for proper TFL1 expression. To confirm binding sites of SVP a set of target genes containing predicted binding sites at the 3' end was selected and analyzed in detail. This set included AGL24, SEEDSTICK (STK), APETALA3 (AP3), and FLOWERING LOCUS C (FLC). As shown in Figure 1c, these genes show peaks of enrichment in the inflorescence ChIP-seq data near their 3'UTR regions and, for $S T K$ and FLC, these regions correspond to predicted SVP binding sites (3'UTR is indicated by the striped rectangle). The enrichments on the 3' UTR were analyzed in independent ChIP-qPCR assays confirming that binding at the 3'UTR is significant (Figure 1d).

Candidate target genes were then identified by associating each gene with an overall $P$ value calculated from the product of the $P$ values of the single binding regions located across the whole gene, encompassing the $3 \mathrm{~kb}$ upstream of the transcription start site to $1 \mathrm{~kb}$ downstream of the transcribed region. Thus, genes could be ranked according to the overall $P$ values obtained. Starting from the ranked gene lists, we selected as high-confidence targets 2,982 genes in seedlings (with a cumulative gene $P$ value < 1.26E-23) and 2,993 genes in inflorescences (cumulative gene $P$ value $<3.16 \mathrm{E}-15$ ) (Additional data file 2, Table S2). The cut-offs on these lists were selected to maximize the number of known targets while excluding the maximum number of genes that were demonstrated to be false positives based on validations with ChIP-qPCR.

\section{Binding motifs of the SVP protein}

MADS-domain proteins are known to bind to different CArG box sequences, including the SRF-type (CC[A/T $]$ 6GG), the MEF2-type (C[A/T]8G), and other intermediate motifs (CC[A/T]7G/C[A/T]7GG) [10,43-46]. In order to assess the enrichment of CArG box motifs within the binding regions obtained from ChIP-seq, and to determine whether there is a preferred form of CArG box for 


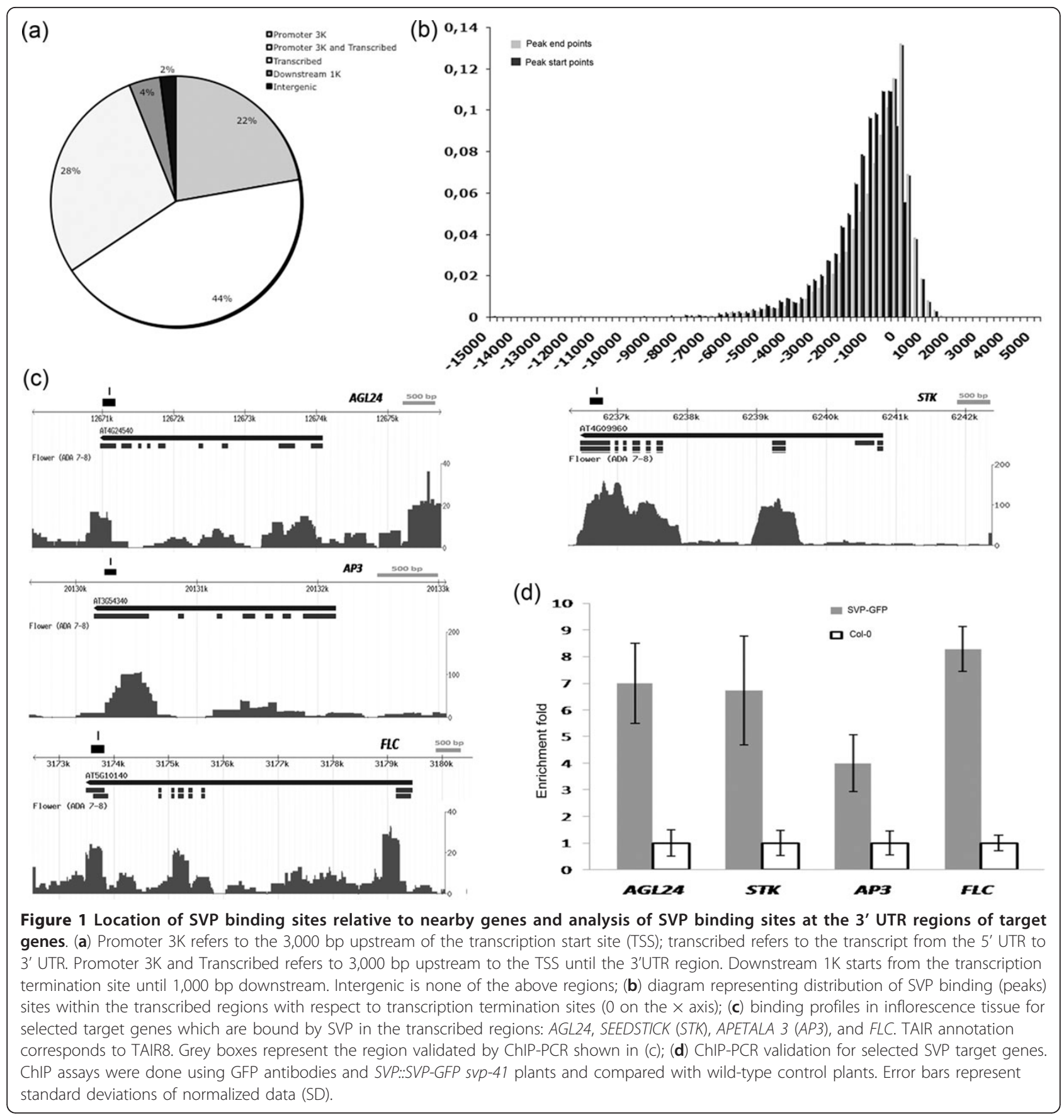

SVP, we ran a tailored version of the motif finder Weeder [47] in order to evaluate separately the enrichment within the regions of each oligonucleotide which could be considered a valid instance of a CArG box given the consensuses described before and also including NC[A/T]6GN. Oligonucleotides found to be enriched in the regions were then clustered together to form the motif maximizing the enrichment score. Motif enrichment was computed according to the Weeder score, which compares the number of occurrences within the ChIP enriched regions to an expected value derived from its number of occurrences genome-wide, computing a log ratio of the fold enrichment. The results are summarized in Figure 2a, split with respect to the two experiments performed and to the ranking of the ChIP regions according to their enrichment $P$ value (best 1,000 regions, best 2,000, and so on). Enrichment clearly increases according to peak rank, with higher CArG box enrichment to be found within the peaks more enriched in the ChIP-seq experiments. Enrichment seems to be slightly higher in flower-enriched 


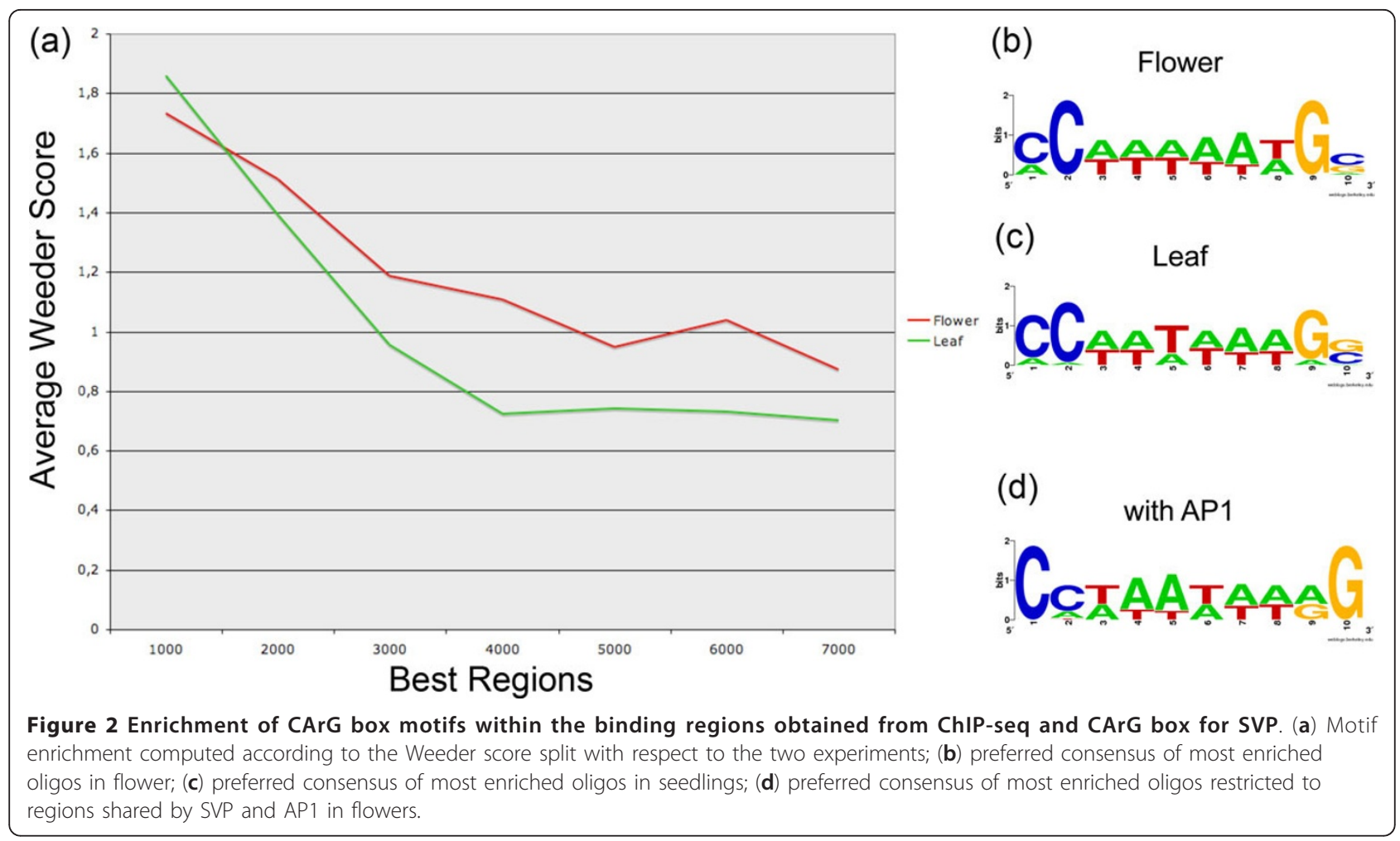

regions with respect to leaf-enriched regions. Also, sequence alignment of most enriched oligos in flowers shows $\mathrm{NC}[\mathrm{A} / \mathrm{T}] 6 \mathrm{GN}$ (shown in the sequence logo of Figure $2 \mathrm{~b}$ and $2 \mathrm{c}$ ) as a preferred consensus, which differs slightly from the already known forms briefly discussed above but closely resembles the one presented in Tao et al. [37]. Finally, oligo analysis restricted to regions shared by SVP and AP1 shows a more canonical CArG box, which is present in the regions with a much higher enrichment (about eight-fold enrichment with respect to the four-fold enrichment in the other regions; Figure 2d).

\section{Comparison of SVP binding behaviour during vegetative and reproductive stages}

During the vegetative stage SVP acts as a repressor of the floral transition $[6,8,18,19]$, while later it plays an important role during floral meristem specification and organogenesis by regulating expression of organ identity genes $[20,25,48]$. Here SVP binding sites were identified in seedlings and inflorescences to compare its behaviour at these two stages. A small number of direct target genes of SVP were previously identified in both vegetative and reproductive tissues $[6,18,24]$. Binding of SVP to these known sites was confirmed in the ChIP-seq data in both conditions, although in some cases (for example SOC1 in vegetative tissues, see below) the enrichment after the IP was not sufficient to exceed the $P$ value threshold employed.
The high confidence lists of putative targets of SVP in vegetative and reproductive tissues show a significant overlap, even if this does not imply a perfect overlap of binding regions for common target genes, as shown in the next section. In total 689 genes appear in both lists, which represents a highly significant overlap $(P$ value $<1 \mathrm{E}-200)$ (Figure 3a and Additional data file 2, Table S2). The GO analyses reveal that the biological processes enriched in both stages are related to development, cell cycle, and DNA metabolism. These may define a set of genes that reflect the core role of SVP during plant development (Figure 3b).

\section{SVP directly binds to flowering-time genes of different regulatory pathways}

Mutations in SVP cause early flowering, illustrating a role for SVP in repressing the floral transition, a process controlled by several regulatory pathways $[6,8]$. Consistent with this function, GO terms related to development, such as 'reproduction' and 'flower development', are significantly overrepresented in the list of putative SVP targets (Figure 3). Moreover, SVP represses flowering by reducing the mRNA levels of FT and TSF $[6,19]$ key components of the photoperiodic pathway, and of the floral integrator SOC1 [18]. In the ChIP-seq data, FT is indeed bound by SVP, but with a low $P$ value $\left(9.5 \times 10^{-7}\right)$ (data not shown). Similarly, ChIP-chip experiments performed by Tao and collaborators were not sensitive enough to detect the binding of SVP to the FT locus [37]. Recent 
(a)

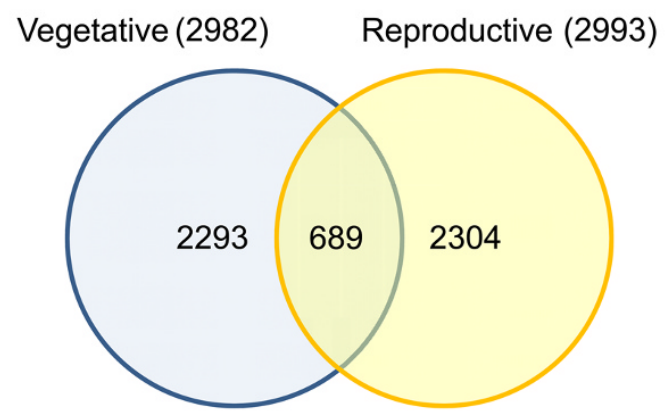

Common target genes

$\mathrm{P}$-Value $<0.001$ (b)

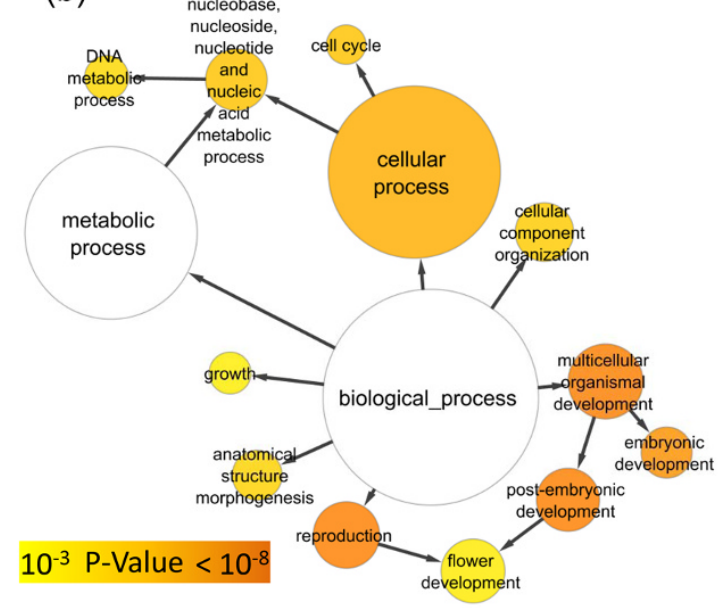

(c)

\section{Vegetative \\ P-Value $<0.001$}

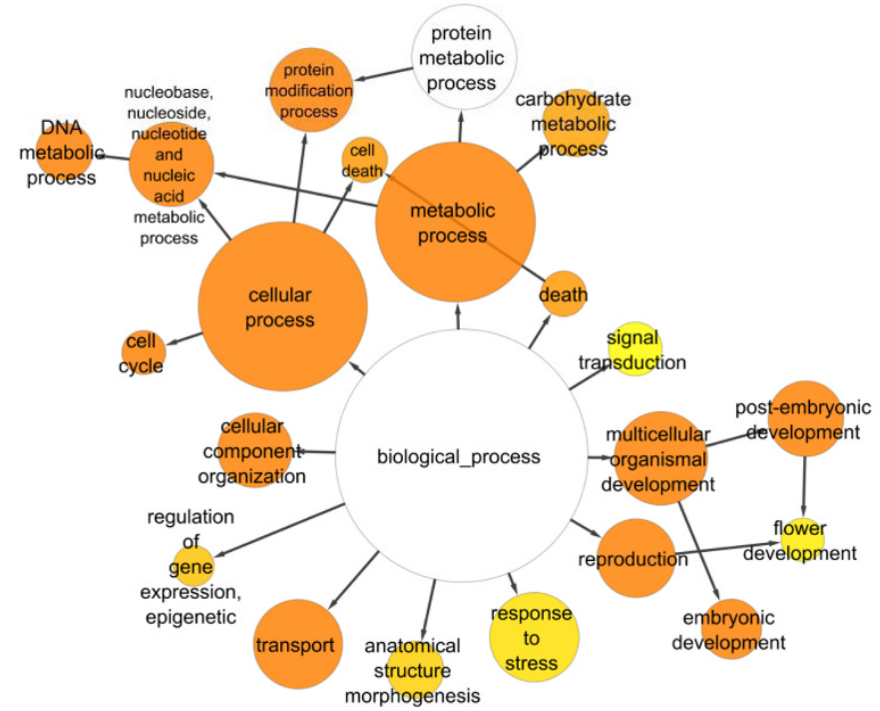

\section{Reproductive \\ P-Value $<0.001$}

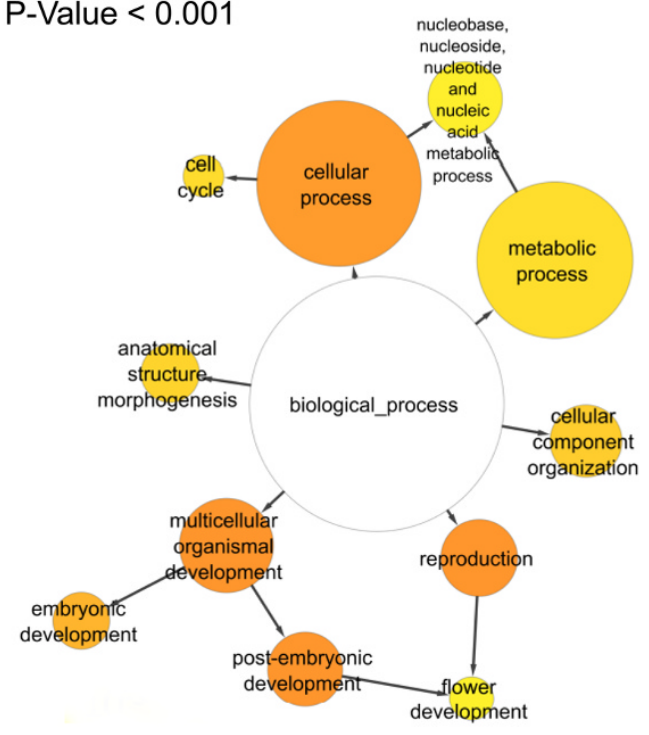

$10^{-3}$ P-Value $<10^{-8}$

Figure 3 Common targets of SVP in vegetative and reproductive tissues and GO enrichment analysis of targets of SVP in vegetative and reproductive tissues. (a) Overlap between high confidence targets of SVP in vegetative and reproductive tissues; (b) GO enrichment analysis of biological process for the common targets of SVP in both tissues. Significant enrichments in the dataset were highlighted in color, different colors represented different levels of significance. The FDR was set up to 0.001 and 0.05 ; (c) visualization of the GO terms related to biological processes enriched in the high confidence lists of targets of SVP in vegetative (left) and reproductive (right) tissues. The color scale represents the level of significance, in terms of FDR, for each category.

work demonstrated that SVP also regulates flowering time independently of $F T$ and SOC1 [18,19]. Thus, we searched the list for known flowering-time regulators. Surprisingly, SVP bound genes involved in several different pathways (Additional data file 1, Table S3), including the circadian clock and photoperiodic pathway, represented by GIGANTEA (GI) and PSEUDO-RESPONSE REGULATOR 7
(PRR7), the autonomous pathway, represented by genes such as FLOWERING LATE KH MOTIF (FLK) and FLOWERING LOCUS D (FLD), genes encoding components of chromatin associated complexes, such as CURLY LEAF (CLF), SWINGER (SWN), and VERNALIZATION2 (VNR2), and the light signaling pathway represented by $P H Y T O-$ CHROME A (PHYA). 


\section{SVP and the regulation of growth regulator signaling during vegetative development}

Growth regulators play different roles in flowering-time control and their molecular links to floral homeotic genes have been extensively reported [13,31,32]. SVP targets related to growth regulator signaling, response, transport and metabolism were identified in the ChIPseq data (Additional data file 3, Table S4). For example, SVP binds directly to STIP (STIMPY), which was recently described as a component of the cytokinin (CK) signaling pathway [49], during the vegetative phase. The expression levels of this gene were tested in $s v p-41$ mutants and Col-0. The qRT-PCR experiments showed that STIP mRNA was present at significantly higher levels in svp-41 mutants compared to Col-0 at all time points tested (Figure 4a). We also quantified the expression levels of STIP mRNA in ft-10 tsf-1 svp-41, which harbours null alleles of $F T$ and TSF and SVP [19]. In $f t$ 10 tsf- 1 svp-41 the expression levels of STIP were upregulated compared to $f t-10$ tsf- 1 double mutants and Col-0 wild-type (Figure $4 \mathrm{~b}$ ), indicating that SVP controls this gene independently of the FT TSF photoperiodic signals. The effect of SVP on STIP expression might indirectly influence the expression of other genes involved in cytokinin signaling. To investigate this possibility a transcriptome analysis was performed by hybridizing RNA extracted from seedlings of wild-type Col-0 and $s v p-41$ to Affymetrix tiling arrays. The results of these experiments demonstrated that 1,381 genes were differentially expressed (FDR $\leq 0.05$ ) in $s v p-41$ compared to Col-0 seedlings (Additional data file 4, Table S5). For some of these genes the change in expression in $s v p-41$ compared to Col-0 was also confirmed by qRT-PCR (Additional data file 1, Figure S2). A GO term test indicated that there is a significant enrichment of genes included in the category 'response to hormonal stimuli' (Additional data file 1, Figure S3 and Table S6). Interestingly seven genes upregulated in $s v p-41$ mutant were related to cytokinin signaling (Figure 4c). These genes belong to two different groups of cytokinin response genes: the type-A ARABIDOPSIS RESPONSE REGULATORS (ARRS) and the CYTOKININ RESPONSE FACTORS $(C R F s)$. These two groups of genes are also transcriptionally activated by STIP [49], suggesting that the control of STIP by SVP has a broad effect on the cytokinin signaling pathway. Indeed, the effect of SVP on CK signaling was also reflected by the significant overlap $(P$ value $=6.6 \times$ $10^{-13}$ ) between the lists of differentially expressed genes in svp-41 mutant and the available expression-profiling data of seedlings treated with the CK benzyladenine (BA) [50] (Figure 4d and Additional data file 5, Table S7).

The ChIP-seq and tiling array data also suggested links between SVP and other growth regulators. For instance, SVP bound several genes involved in auxin signal transduction, such as $B I G$, which encodes a putative auxin transporter required for normal auxin efflux and inflorescence development (Additional data file 3, Table S4) $[51,52]$. Another gene bound by SVP is CORONATINE INSENSITIVE 1 (COI1), which encodes the jasmonate receptor (Additional data file 3, Table S4) [53,54]. Therefore SVP might affect auxin and jamonate homeostasis by directly binding to genes encoding key components of their signaling cascade pathways. In agreement with this conclusion, our Tiling array data showed that members of the SAUR-like auxin-responsive family were up-regulated in svp-41 mutant (Additional data file 3, Table S4 and Additional data file 1, Figure S2). In addition, six of the JASMONATE ZIM-domain (JAZ) genes (JAZ1, 5, 6, 7, 8, and 10 ), which are part of the jasmonate signaling pathway and are transcriptionally activated by the hormone, were increased in expression in the mutant compared to Col-O (Additional data file 3, Table S4 and Additional data file 1, Figure S2).

\section{Common targets of SVP and FLC during vegetative development}

MADS-domain proteins form multimeric complexes that are proposed to be important in determining their DNA binding specificity. Co-immunoprecipitation analysis and yeast two-hybrid assays demonstrated that SVP interacts with the related MADS-domain protein FLC and genetic data indicate that this interaction is likely functionally important in the control of flowering time [18,55]. Moreover, SVP associates with the promoter region of $\mathrm{SOC1}$ and the intron of $F T$ where FLC also binds [18,39]. Recently the genome wide targets of FLC were identified using ChIP-seq technology [32]. Of these FLC putative targets, 112 were also detected in our experiment as being bound by SVP in vegetative tissue $\left(P\right.$ value $\left.=1.9 \times 10^{-6}\right)$ (Additional data file 1, Figure S4a). Nine of the FLC putative targets were previously validated by ChIP-qPCR and six of them shown to change in expression in $f l c-3$ mutants [32]. Of these confirmed FLC targets, four were selected to test by ChIP-qPCR if they were also bound by SVP (Figure 5b, c). Of these four FLC targets, three were bound by SVP in a similar location. One of these was $J A Z 6$, which was bound by FLC in its promoter region and its expression is increased in flc-3 [18]. JAZ6 expression was also upregulated in svp-41 (Figure 5a), however it was not enriched in our ChIP-seq experiment, and this was confirmed by independent ChIP-qPCR analysis, suggesting that the changes in JAZ6 expression caused by SVP are not an effect of direct binding (Figure 5c). A second confirmed FLC target, AGL16, was not enriched in the SVP ChIP-seq data, however the region bound by FLC showed a low but consistent enrichment in ChIPqPCR of SVP. This experiment suggests that SVP is weakly bound to the same region of AGL16 as FLC, and 


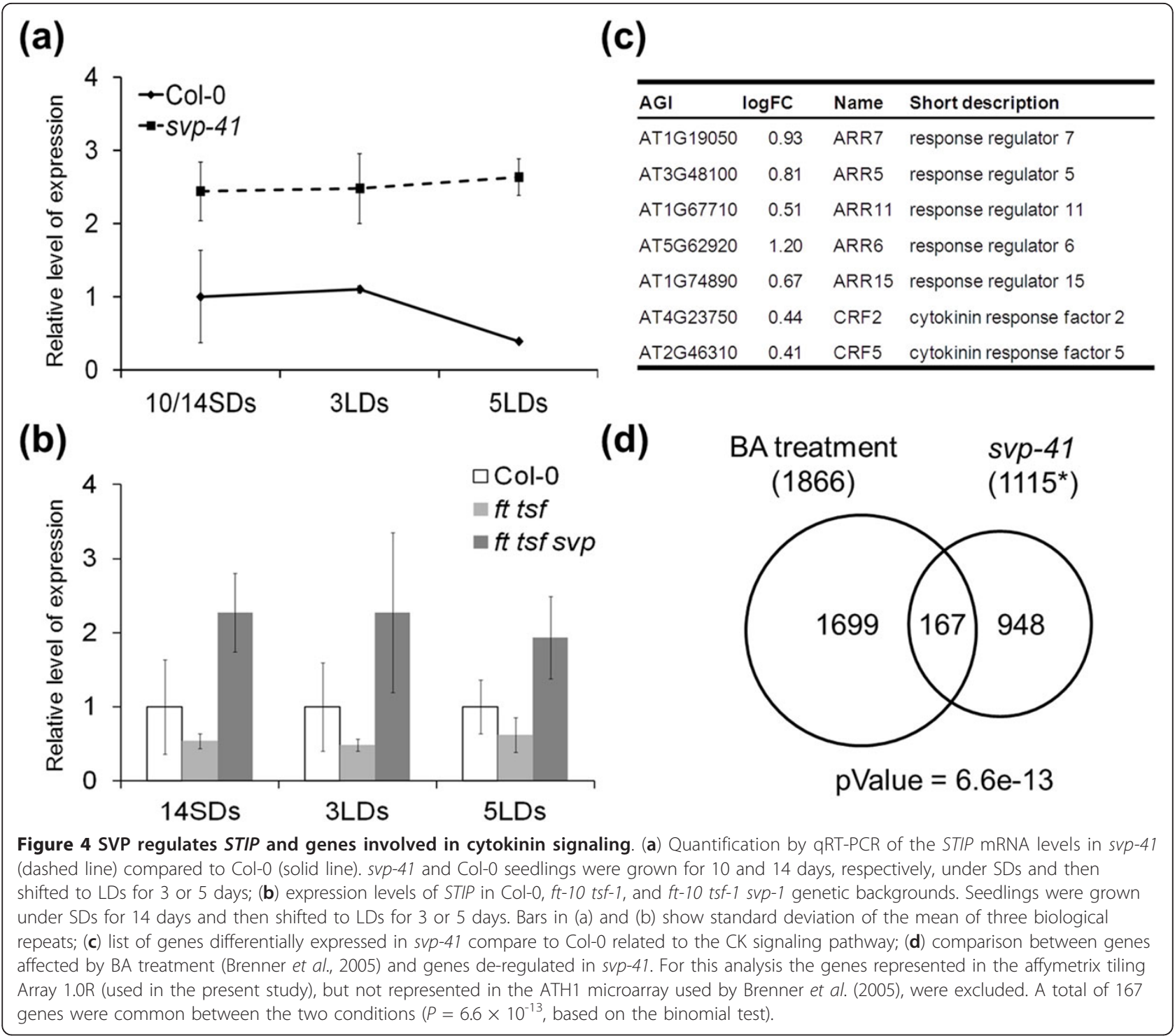

the low enrichment might explain why it was not detected in the ChIP-seq experiment. AGL16 expression was not changed in $s v p-41$ compared to Col, similar to what was observed in $f l c-3$. A third confirmed FLC target was SVP, and ChIP-qPCR confirmed that SVP binds to the same region in its own promoter as FLC. These ChIP-qPCR experiments demonstrate that there is a strong but not complete overlap in the targets of FLC and SVP.

\section{SVP auto-regulates its gene expression in vegetative} tissue and flowers

The ChIP-seq data indicated that SVP binds to its own genomic region in vegetative tissue and flowers. However, regions actually bound in both tissues may differ. This differential binding was confirmed by independent ChIP-qPCR experiments on two specific regions named I and II (Figure 6 a-c), located approximately 2,000 bp upstream of the 5'UTR and in the terminal part of the $S V P$ first intron, respectively. As shown in Figure $6 \mathrm{~b}$ and $6 \mathrm{c}$, SVP binds site I in floral tissue but not in vegetative tissue, whereas site II is bound in both tissues. Whether binding of SVP influenced its own expression was tested in different ways. In addition to the microarray experiment described above, another transcriptome analysis was performed by hybridizing RNA extracted from inflorescences of wild type Col-0 and svp-41 agl24. ap1-12 to affymetrix tiling arrays. In this experiment 246 genes were differentially expressed (FDR $\leq 0.05$ ) in svp-41 agl24 ap1-12 compared to Col-0 inflorescences (Additional data file 4, Table S5). The tiling array expression data showed that SVP mRNA was downregulated in the $s v p-41$ single mutant in vegetative tissues $(\operatorname{logFC}-1.13 ; P=0.001)$ as well as in inflorescences of the $s v p-41$ agl24-2 ap1-12 triple mutant (logFC -0.86; 
(a)

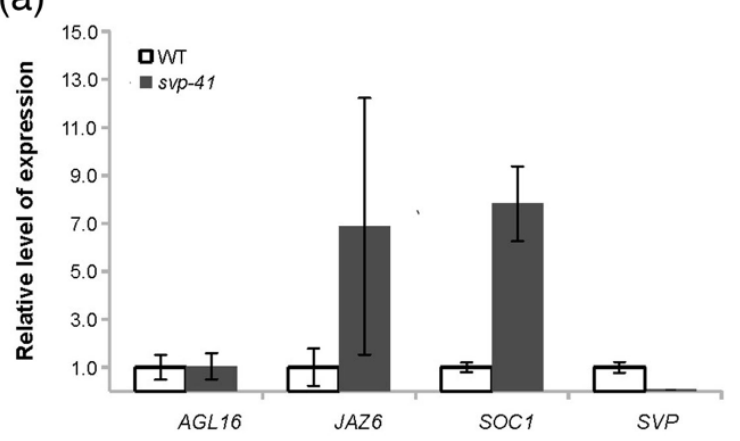

(b)
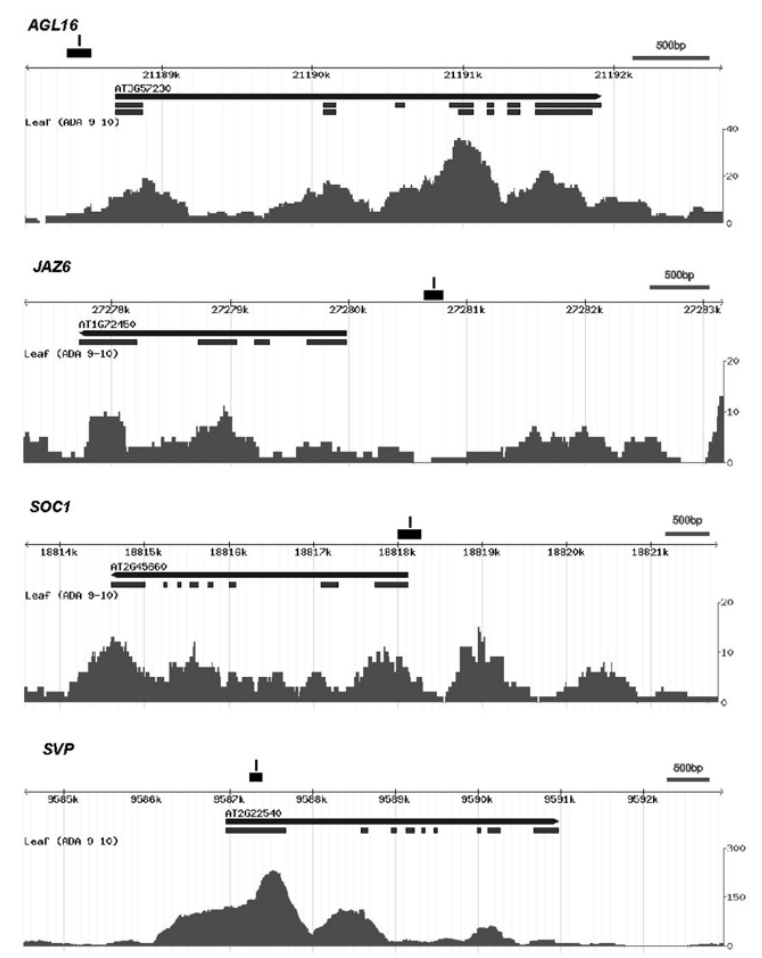

(c)

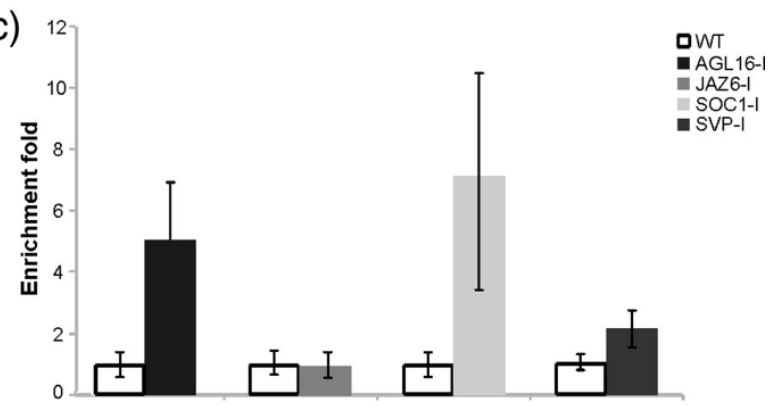

Figure 5 Common targets of SVP and FLC. (a) Expression of known direct targets of FLC in svp-41. Data represent expression of selected genes in microarray experiment with FDR $<0.05$. The expression level of each gene in svp-41 was normalized to the level of wild type Col-0.

Error bars represent SDs of normalized data; (b) binding profiles of ChIP-seq experiment for the selected genes. TAIR annotation corresponds to TAIR8. Grey boxes represent the region validated by ChIP-PCR which are shown in panel (c); (c) ChIP-PCR validation of selected genes using antiGFP antibodies using seedlings of wild type Col-0 and SVP::SVP-GFP sVp-41 lines. Results are expressed relative to actin. Error bars represent SD. 


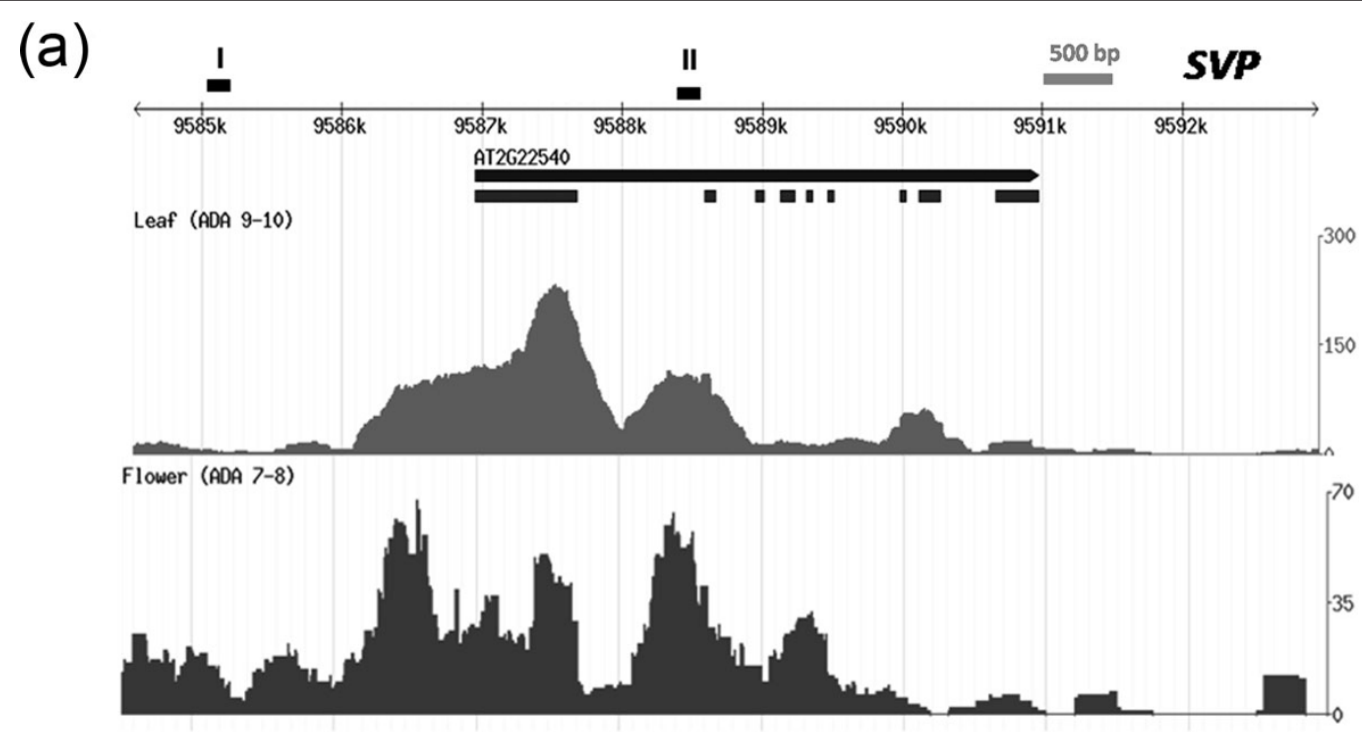

(b)

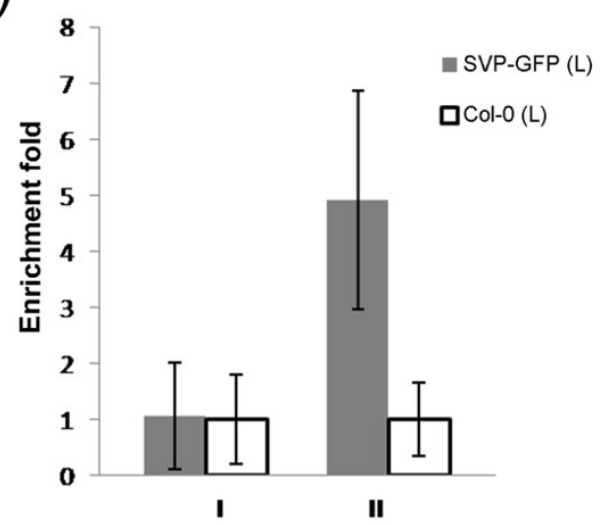

(d)

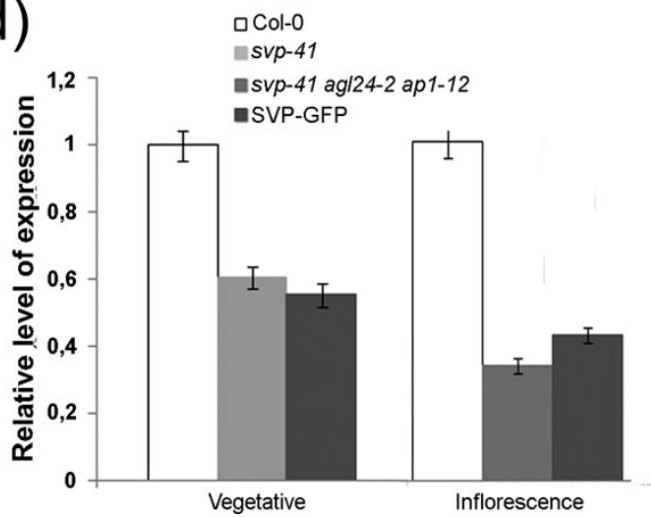

(c)

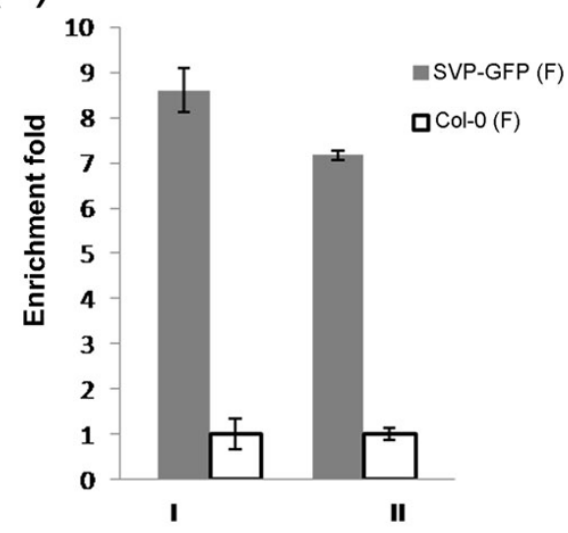

(e)

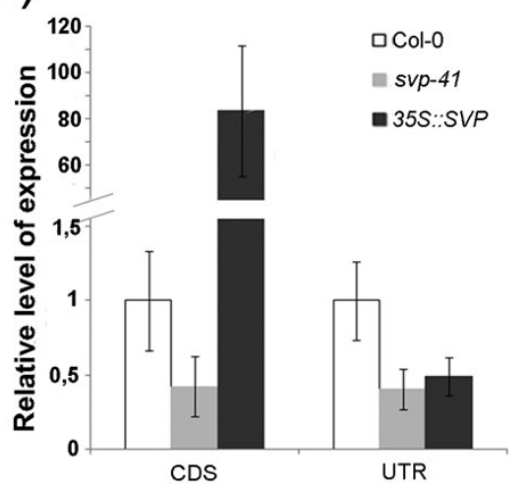

Figure 6 SVP binds and regulates itself. (a) Binding profiles for SVP on SVP genomic locus in seedlings (upper panel) and inflorescence (lower panel) tissues. TAIR annotation corresponds to TAIR8. Grey boxes represent the region validated by ChIP-PCR in panels (b) and (c); (b) and (c) ChIP-PCR validations for two specific regions named I and II. ChIP assays were done using GFP antibodies and SVP::SVP-GFP sVp-41 plants and compared to wild-type control plants. ChIP-PCR validation in vegetative (b) and reproductive tissue (c); (d) qRT-PCR expression analysis using primers for the SVP 3'UTR region. RNA was extracted from wild-type Col-0, sVp-41, and SVP::SVP-GFP svp-41 seedlings and from wild-type Col-0, sVp-41 agl24-2 ap1-12 triple mutant, and SVP::SVP-GFP sVp-41 inflorescences; (e) qRT-PCR expression analyses using primers for the SVP $3^{\prime} U T R$ region and coding region. RNA was extracted from wild-type Col-0, svp-41, and 35S::SVP seedlings. In all graphs error bars represent the standard deviation of normalized data (SD). 
$P=0.02)$. This downregulation was validated by qRT-PCR using independent $s v p-41$ single mutant, svp-41 agl24-2 ap1-12 triple mutant and wild-type cDNA samples obtained from RNA extracted from seedlings and inflorescences (Figure 6d). Since $s v p-41$ is a deletion mutant in which two base pairs are deleted in the second exon resulting in a frame-shift of the open reading frame [8], this reduction in mRNA level might be due to nonsense-mediated decay [56]. To investigate this possibility, we performed qRT-PCR assays using primers designed on the 3'UTR region of the endogenous $S V P$ gene, which is not present in the SVP::SVP-GFP fusion construct. RNA was extracted from wild-type, svp-41 and $S V P:: S V P-G F P$ svp-41 seedlings and from wild-type, svp-41 agl24-2 ap1-12 and SVP::SVP-GFP svp-41 inflorescences (Figure $6 \mathrm{~d}$ ). The results confirmed a reduction in mRNA level also in SVP::SVP-GFP svp-41 tissues suggesting that indeed this reduction in $S V P$ mRNA level seems to depend on the mRNA instability in the mutant background. As an alternative approach the abundance of SVP mRNA expressed from the endogenous gene was tested in plants in which $S V P$ was overexpressed from a $35 S:: S V P$ transgene. A qRT-PCR strategy was used in which the cDNA expressed from the transgene and endogenous gene can be distinguished (Figure 6e). This experiment demonstrated that $S V P$ mRNA expressed from the endogenous locus is reduced in $35 S:: S V P$ plants. Taken together our data suggest that SVP directly regulates its own expression, and that it probably acts to repress its own transcription.

\section{Genes involved in meristem development are targets of SVP at two developmental stages}

Genes involved in meristem development were enriched as SVP targets in both vegetative material and flowers. SVP is expressed in the SAM during the vegetative stage $[6,8,18,19]$. In addition it plays an important role during floral meristem specification and organogenesis $[25,48]$. Consistent with this idea a significant enrichment of SVP target genes related to post-embryonic developmental processes was detected in the ChIP-seq results of both vegetative and reproductive samples (Figure 3c). Due to the expression pattern of $S V P$, putative targets with annotated functions in meristem development were screened for directly (Additional data file 3, Table S4). The $C L V$-WUS feedback loop plays a central role in maintaining meristematic activities [57]. In the ChIP-seq data $C L V 1$ and $C L V 2$, two important players in WUS regulation, are targets of SVP in vegetative tissues and $C L V 1$ is also bound during reproductive development. Additionally, according to the ChIP-seq data, the HDZIPIII encoding genes PHABULOSA (PHB), PHAVOLUTA (PHV), REVOLUTA (REV), and HOMEOBOX GENE 8 (ATHB8), which regulate post-embryonic meristem initiation [58], are also bound by SVP in vegetative tissue. Furthermore, $P H B$ which is a regulator of the size of the WUS-expression domain [59], is also bound by SVP in the floral meristem. In order to test whether the binding of SVP to some of these genes affects their spatial pattern of expression we performed RNA in-situ hybridization experiments. A broader expression pattern of $P H B$ and $C L V 1$ was observed in shoot apical meristems of $s v p-41$ mutants than Col-0 wild-type plants grown for 2 weeks under SDs (vegetative phase) (Figure 7a, b, d, e). However, these differences might be due to the larger size of the $s v p-41$ meristem compared to Col-0 at this stage. Thus, the patterns of expression of $P H B$ and CLV1 were also compared in 10-day-old svp-41 mutants and 2-week-old Col0 plants, which have SAMs of similar size. Confirming our previous result $P H B$ and $C L V 1$ mRNA were detected in a broader region of the $s v p-41$ (10 SDs) SAM compared to Col-0 (Figure 7c and 7f). These results together with the ChIP-seq data suggest that SVP directly regulates the expression pattern of these genes. Furthermore, KANADI1 (KAN1) and KAN2, involved in the establishment of abaxial-adaxial polarity in lateral organs produced from the apical meristem, resulted also to be direct targets of SVP in inflorescences. It has been hypothesized that complementary regions of action of the class III HD-ZIP genes and $K A N A D I$ genes leads to the establishment of adaxial and abaxial domains in developing lateral organs. The possible role of SVP and other MADS-domain proteins in the regulation of part of these genes in reproductive tissues is presented below.

\section{Genome wide targets of SVP during flower development and comparison with the targets of AP1 and SEP3}

During the early stages of flower development (stage 1 and 2) AP1 interacts with SVP and the dimer recruits the SEU-LUG repressor complex to control the expression of homeotic genes to maintain the floral meristem in an undifferentiated state [25]. At late stage 2, when SVP expression is switched off, AP1 interacts with SEP3 to control sepal and petal identity. Recently, genomewide binding studies for SEP3 and AP1 during inflorescence development were published [13,31] providing the opportunity to compare these datasets with the one obtained here for SVP.

A total of 265 common putative targets for both SVP and AP1 were identified ( $P$ value $<7.2 \mathrm{E}-06$ ) (Additional data file 6, Table S8 and Additional data file 1, Figure S4). This overlap is expected because SVP and AP1 act redundantly during floral meristem specification where their expression domains overlap [24]. Interestingly transcription factors are enriched among common targets. In addition SVP binds to AP1, suggesting that it regulates a 


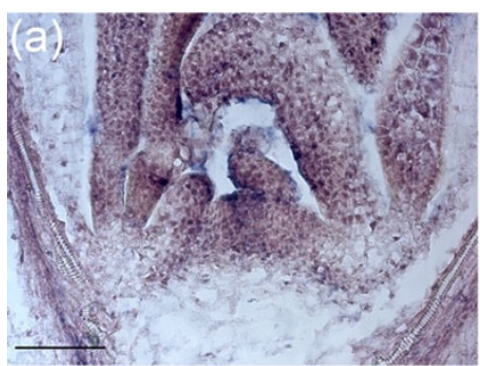

(d).
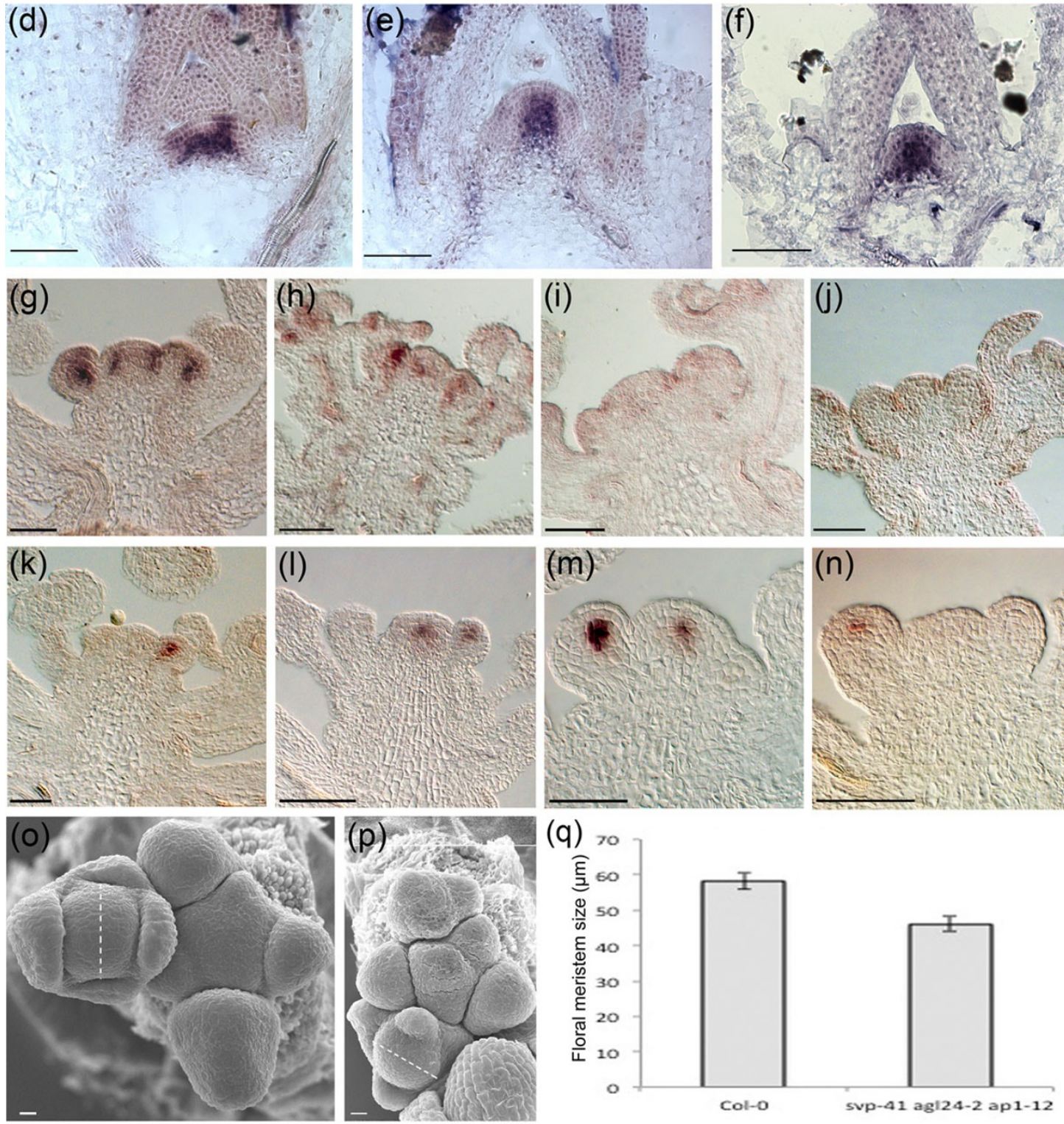

Figure 7 Expression analysis of meristem developmental genes by in-situ hybridization analysis in vegetative and reproductive tissues and floral meristem size analysis. (a-c) Patterns of expression of PHB: (a) 14-day-old wild-type, (b) 14-day-old svp-41, and (c) 10-day-old svp-41 mutant; (d-f) patterns of expression of CLV1: (d) 14-day-old wild-type, (e) 14-day-old svp-41 mutant, and (f) 10-day-old svp-41 mutant; in both svp-41 10 and 14-day-old seedlings the PHB and CLV1 mRNA were detected in a broader region of the SAM compared to Col-0; (g, h) expression of ARF3 in wild type and svp-41 ag/24-2 ap1-12 inflorescence respectively; (i, j) KAN1 expression pattern in wild-type and svp-41 agl242 ap 1-12 inflorescences; (k, I) CLV1 expression in wild-type and svp-41 ag/24-2 ap1-12 inflorescence; $(\mathbf{m}, \mathbf{n})$ expression profile of WUS in wild-type and svp-41 agl24-2 ap 1-12 inflorescences, its expression seems to be higher in the wild-type FM than in the triple mutant FMs at the same developmental stage. The scale bar represents $50 \mu \mathrm{m}$. (o) View of wild-type inflorescence; (p) view of svp-41 agl24-2 ap 1-12 inflorescences; central zone of triple mutant FMs at stage 3 were compared to those of wild-type plants. The scale bar represents $10 \mu \mathrm{m}$. (q) Diagram showing the difference in FMs size between the wild-type and svp-41 agl24-2 ap 1-12 triple mutant central dome, error bars represent standard error (SE). 
functionally redundant gene as well as itself. The overlap between the targets of SVP with those published for SEP3 [13] revealed 413 ( $P$ value $<5.91 \mathrm{E}-10)$ genes that are bound by both of these MADS domain transcription factors (Additional data file 6, Table S8 and Additional data file 1, Figure S4). KAN1, CLV1, PHB, and ARF3 also named ETTIN, that are present in the subset of genes bound by SVP and AP1, are also present in the list of genes regulated by both SVP and SEP3.

Transcriptome data obtained from the tiling array hybridization experiments using RNA extracted from inflorescences of Col-0 and the svp-41 agl24 ap1-12 mutant showed that the number of differentially expressed genes were considerably fewer than those found by comparing the vegetative tissue of $s v p-41$ and Col-0 wild-type plants (Additional data file 4, Table S5). However, the number of deregulated genes might be underestimated in this analysis because the whole inflorescence of svp-41 agl24 ap1-12 mutant plants were used, whereas $S V P$ expression is restricted to stage 1-2 FMs only. Therefore, altered expression of several targets might not be detected in this material. To overcome this we also checked the expression of putative SVP target genes by a qRT-PCR approach, collecting the most inner parts of Col-O and svp-41 agl24 ap1-12 inflorescences, avoiding the already opened flowers. Both KAN1 and PHB mRNAs were increased in abundance in the svp-41 agl24-2 ap1-12 mutant background compared to wild-type (Figure 8a) and the enrichment of these genes observed in the ChIP-seq experiment was confirmed by means of independent ChIP-qPCR analysis (Figure $8 \mathrm{~b}$ and $8 \mathrm{c}$ ), suggesting a direct regulation of these genes by both SVP and AP1 during flower development. Interestingly also $C L V 1$, which plays an important role in establishing and maintaining floral meristem identity [60], is a direct target of both SVP and AP1 in reproductive tissue and its expression was increased in the svp-41 agl24-2 ap1-12 triple mutant compared to wild-type (Figure 8a-c). Another transcription factor encoding gene that is bound by SVP and AP1 and upregulated in svp-41 agl24-2 ap1-12 inflorescences is ARF3 (Figure $8 \mathrm{a}-\mathrm{c}$ ). ARFs are proteins that are activated by convergent auxin flow. Dynamic changes in auxin fluxes are mediated by PIN proteins and interestingly SVP and AP1 can interact with the genomic region of PIN1. Analysis by qRT-PCR showed increased levels of PIN1 mRNA in svp-41 agl24-2 ap1-12 inflorescences in comparison to the wild-type control, suggesting a direct role of SVP and AP1 in its regulation which was confirmed by independent ChIP-qPCR experiments (Figure 8a-c). We further examined the expression of $A R F 3, C L V 1$, $K A N 1, P H B$, and PIN1 in response to SVP activation using the functional steroid-inducible system. The svp41 agl24-2 ap1-10 triple mutant was transformed with a construct in which the $35 \mathrm{~S}$ promoter directs a fusion between SVP and a part of the rat glucocorticoid receptor (GR), as reported previously [61]. The svp-41 agl242 ap1-10 mutant forms cauliflower like curds since its unable to establish FM identity and therefore it proliferates IMs instead. The obtained transgenic plants showed upon induction with the steroid dexamethasone (DEX) rescue of the development of FMs and flowers that resembled those of the agl24-2 ap1-10 double mutant (Additional data file 1, Figure S5). We treated the inflorescences twice, at time 0 and again after $8 \mathrm{~h}$ with DEX and collected the material after $24 \mathrm{~h}$ from the first treatment. This time point was selected according to Smyth et al. [40], since they showed that the duration of stage 1 of flower development is $24 \mathrm{~h}$. ARF3, CLV1, KAN1, $P H B$, and PIN1 expression levels were all decreased after DEX treatment of svp-41 agl24-2 ap1-10 35S::SVPGR inflorescences, confirming that SVP acts as a repressor of those genes (Figure 8d).

To investigate the changes in expression profiles of some of these target genes, we performed in-situ hybridization experiments using wild type and svp-41 agl24-2 ap1-12 inflorescences (Figure 7g-n). For ARF3, KAN1, and $C L V 1$ the expression pattern was not changed suggesting that the upregulation of these genes is not due to ectopic expression. Interestingly in situs using a specific probe for WUS clearly showed that in comparison to wild-type, in stage $2 \mathrm{FMs}$ this gene was lower expressed in the $s v p-41$ agl24-2 ap1-12 triple mutant. Since svp-41 agl24-2 ap1-12 flowers show reduced numbers of floral organs compared to wild-type or any of the single mutants [25], we wondered if these defects were caused by changes in meristem size. Therefore the central zone of FMs at stage 3 of flower development of the svp-41 agl24-2 ap1-12 triple mutant and wild-type were compared. The size of the central zone is defined by the distance between the opposite lateral sepals (Figure 7o-q). The svp-41 agl24-2 ap1-12 FMs were significantly smaller, as compared to those of wild-type plants (Table 1 and Figure 7q). Taken together all these data suggest a role of SVP in the control of FM size, probably by modulating the expression of genes involved in the CLV-WUS pathway.

\section{SVP binds in reproductive tissues to genes encoding post-translational regulators}

Interestingly, the high confidence list of SVP target genes in inflorescence tissue exhibits a significant enrichment of genes related to Cullin-RING ubiquitin ligase complexes, mainly involved in post-translational regulation of substrate proteins by attaching poly-ubiquitin chains that target the substrate for $26 \mathrm{~S}$ proteasome degradation $[62,63]$. The substrate specificity of CUL4-RING-LIGASES (CRL4s) is exerted by proteins that contain a DWD box (DDB1-binding WD-40 box) 


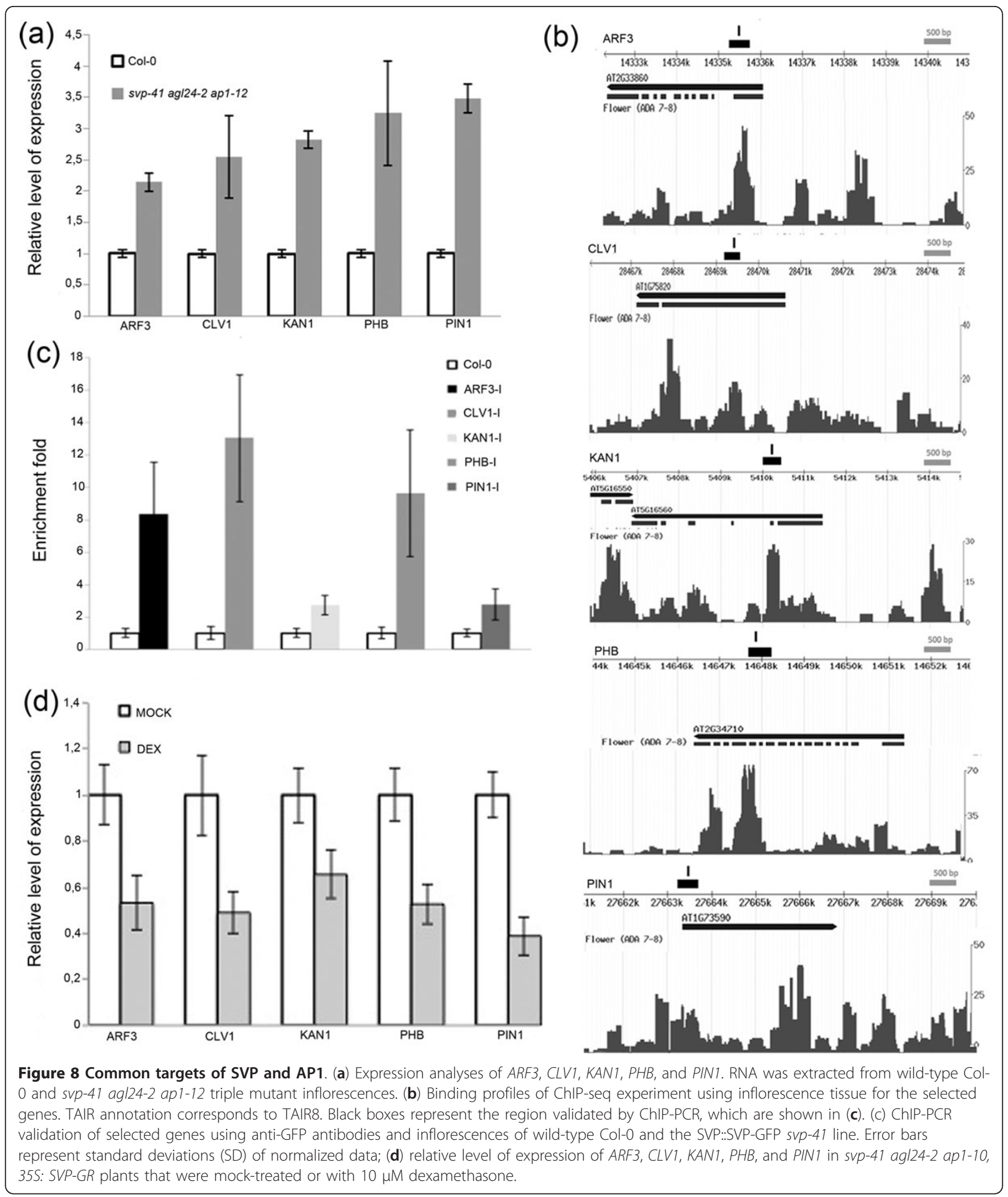

or a WDxR sub-motif [64-67]. Proteins with these motifs are referred to as potential DCAF (DDB1-CUL4 ASSOCIATED FACTOR) proteins [67], which may target proteins for ubiquitinilation $[64,68]$. However, they have also been implicated in chromatin mediated transcriptional control [69]. In Arabidopsis, 119 different putative DCAF proteins have been identified [67] and our ChIP-seq experiments suggest that nearly half of 
Table 1 Floral meristem size

\begin{tabular}{ll}
\hline & Floral meristem mean \pm SE $(\mu \mathrm{m})$ \\
Col-0 & $n=858.1 \pm 2.2$ \\
svp-41 agl24-2 ap 1-12 & $n=846.7 \pm 2$ \\
\hline
\end{tabular}

Col-0 vs. svp-41 agl24-2 ap1-12: Two sample T-test, $t=3.9200, D F=14$, $P=0.0015$.

them (47 of 119) are targets of SVP in both tissues tested and more than half of these (26 of 47) are putative SVP targets in reproductive tissues (Additional data file 1, Table S9).

Among the putative DCAF floral SVP targets to which a function in floral development had not previously been ascribed (Additional data file 1, Table S9), we selected WDR55 as a case study for detailed analyses of its function as a SVP target in flower development.

\section{The regulation of WDR55 by SVP forms as an alternative pathway for the regulation of $A G$}

WDR55 was shown to interact with DDB1A, suggesting a regulative role through a putative CUL4-DDB1 WDR55 E3 complex, and plays a major role in Arabidopsis reproductive development. WDR55 is required for gametogenesis and embryogenesis and is suggested to be involved in auxin-dependent regulation of embryo development [70].

In order to verify that WDR55 expression requires $S V P$, we performed qRT-PCR analyses on $s v p$ double and triple mutant combinations. Compared to wild-type, WDR55 transcripts were reduced in abundance in the double mutant svp-41 agl24-2 $\left(30^{\circ} \mathrm{C}\right)$ and in the svp-41 agl24-2 ap 1-12 mutant background (Figure 9a). The binding of SVP to WDR55, as observed in the ChIP-seq experiment, was confirmed by means of independent ChIP-qPCR analysis (Figure 9b), suggesting that changes of WDR55 expression in svp-41 agl24-2 and svp-41 agl24-2 ap1-12 are due to the direct action of SVP during flower development.

A recent report describes two mutant alleles of WDR55 that demonstrate a requirement of WDR55 in gametophyte development and function, as well as for setting up the embryo body plan. The weaker of these alleles, $w d r 55-2$, displayed close to mendelian ratios of mutant seeds $(22.7 \%)$ and no homozygous plants could be identified, although a small fraction (2\%) could be expected from the genetic data [70]. In order to screen for the theoretical presence of homozygous plants in the progeny, we allowed a large number of seeds from heterozygous $w d r 55-2$ plants to germinate for a prolonged period on MS-2 agar plates containing glufosinate (BASTA) selection. Indeed, we identified a class of late germinating, small seedlings that initially were smaller than the glufosinate sensitive seedlings $(3.6 \%, n=1,035)$. However, this class was BASTA resistant and thus carried the wdr55-2 mutation.

Generally, wdr55-2 seedlings supported growth, but were severely delayed compared to wild-type. In particular, wdr55-2 inflorescences were smaller than wild-type and had fewer flowers. Upon inspection we found that the mutant floral organs were generally smaller and often morphologically distinct from wild-type (Figure 9 and Additional data file 1, Figure S6). The sepals were thinner and often fused at early stages and did not separate completely at maturation (Figure 9d and 9e, Additional data file 1, Figure S6b, c and Table S10). The petals were smaller and thinner, as well as being non-uniform in size (Additional data file 1, Figure S6e and Table S10). The stamens were smaller and never occurred in sixes as in wild-type Col (Additional data file 1, Figure S6 and Table S10). The $w d r 55-2$ flowers also displayed homeotic transformations (Figure 9g, i). We observed unfused carpels (Figure 9f), carpeloid sepals (Figure 9g), petals that resemble stamens filaments and carpeloid filaments with ectopic papillar cells (Figure 9i) at a moderate frequency. New flowers appeared to grow out from whorl 1 or 2 at a low frequency (Figure 9j) and most of the flowers appeared to be asymmetric in flower organ organization (Figure 9h).

Due to the homeotic transformations observed in $w d r 55-2$ flowers, we checked the expression of the organ identity genes APETALA3 (AP3), PISTILLATA (PI), and AGAMOUS (AG) by in-situ hybridization (Figure 9k-n and Additional data file 1, Figure S7). The in-situ analysis shows that in the $w d r 55-2$ mutant, the expression pattern of both AP3 and PI is maintained as wild-type plants (Additional data file 1, Figure S7).

$A G$ is expressed in the inner part of the floral meristem where stamen and carpel primordia develop. During flower development $A G$ expression is restricted to whorls 3 and 4 (Figure 9k). The in-situ analysis shows that in the $w d r 55-2$ mutant, $A G$ is expressed in chimeric organs that develop in the second whorls (Figure 9l) as well as in carpelloid-sepals developing in first whorls (Figure 9m) where stigmatic tissues and carpelloid structures are detectable. $A G$ is expressed already in early stages of flower development, in particular stage 1 (Figure 9n), but the architecture of inflorescences in $w d r 55-2$ makes precise staging difficult.

SEU, LUG, AP1, and SVP are involved in $A G$ regulation, and by mutation ectopic $A G$ expression is found [25,71-73]. SEU and LUG are thought to be cadastral genes, and are involved in the control of expression boundaries of floral homeotic genes [71,73] and they interact to repress AGAMOUS $(A G)$ in the outer two whorls of the flower [72,73]. The SVP-AP1 dimer binds the LUG-SEU repressor and directly regulates $A G$ expression during early stages of flower development 


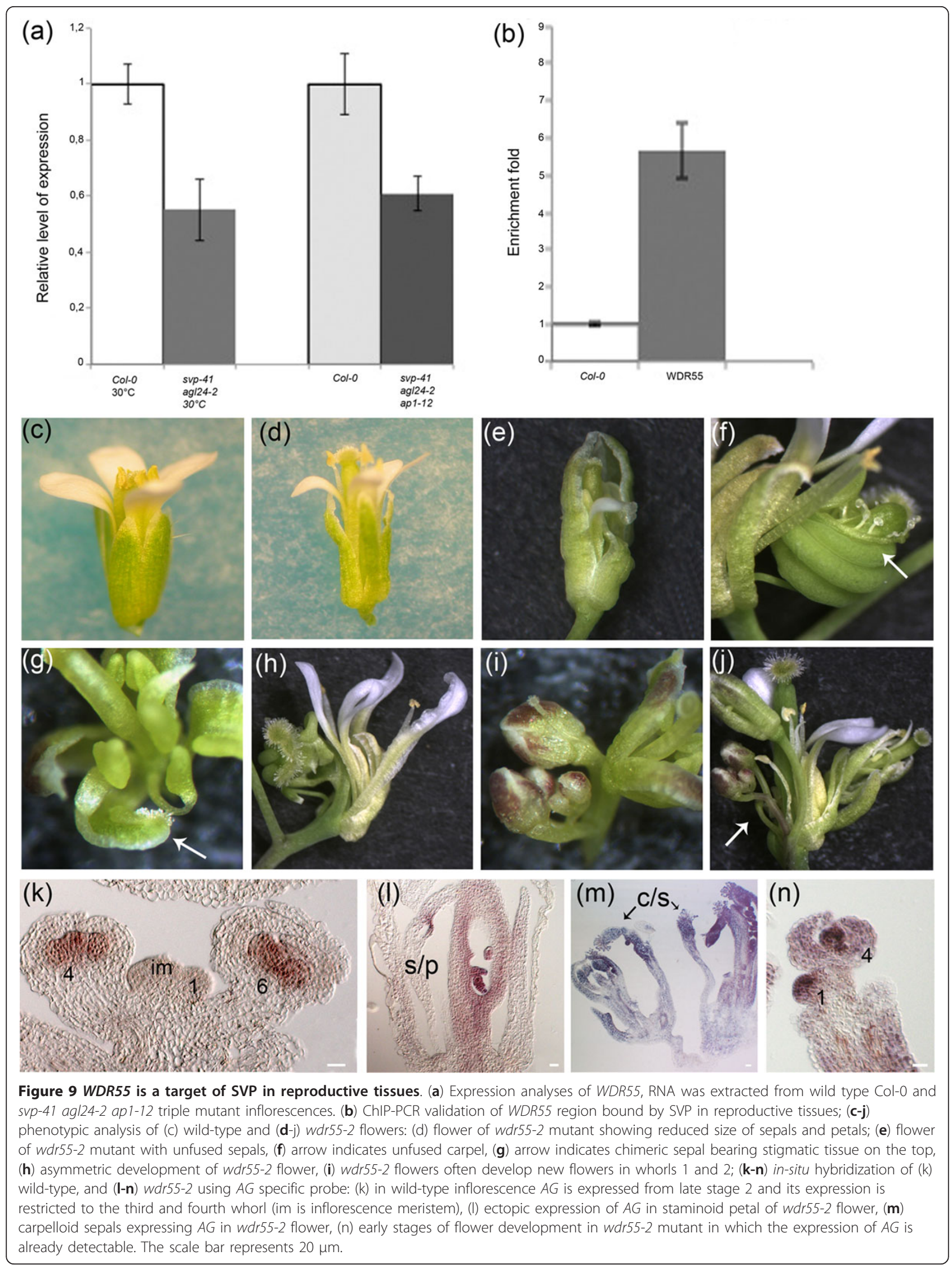


[20,25]. To investigate the regulation of $A G$ through WDR55 further, a Yeast-2-Hybrid ( $\mathrm{Y} 2 \mathrm{H})$ was performed with SEU, LUG, AP1, and SVP. Upon repeated testing, however, WDR55 did not interact with any of these proteins (data not shown). This could be due to weak interactions, and thus not detectable in our $\mathrm{Y} 2 \mathrm{H}$ system, or WDR55 does not directly interact on a protein level with these $A G$ regulators.

Taken together, our data suggest a role of WDR55 in floral development. In particular it seems to control the pattern of $A G$ expression independently from LUG-SEU repressor complex, indicating an additional pathway by which SVP repress $A G$ expression. However, the function of WDR55 in flowers does not seem to be restricted to the regulation of the boundaries of $A G$ expression as exemplified by the ag- $1 w d r 55-2$ double mutant (Additional data file 1, Figure S8).

\section{Discussion}

The MADS-domain factor SVP has different functions during development. An 'early' function as a repressor of the floral transition and a 'later' function in floral meristem identity specification $[6,8,18,20,24,25,48]$. These two functions are also reflected by $S V P$ expression, which is present in the leaves and SAM during the vegetative phase, is repressed in the meristem when plants switch to reproductive development and then reappears in the floral meristem during the early stages of flower development $[8,24]$. Whether SVP regulates different or similar sets of genes during these two phases of development is unknown. We employed ChIP-seq analysis to study the genome-wide binding behavior of SVP during these phases. SVP was found to bind to approximately 3,000 genes at both stages of development. Some genes were regulated by SVP at both stages of development, such as those in pathways regulating meristem development, whereas others were specific to one of the stages. One mechanism by which these differences in target gene specificity are likely to occur is through interactions between SVP and other MADS domain protein partners generating complexes with different specificities. Consistent with this idea, comparison of the targets of SVP and two of its partners, AP1 and FLC, showed similarities and differences.

\section{Genome-wide ChIP-Seq experiments reveal several roles for SVP in modulating vegetative development}

SVP bound to approximately 3,000 genes during vegetative development. GO terms analysis of these genes identified functional categories such as 'reproduction' and 'flower development' as being significantly over-represented in the list of putative SVP targets (Figure 3c). Similar results were previously found by Tao et al. [37]. These authors performed ChIP-chip experiments and identified a total of
328 genes bound by SVP during floral transition [37]. Comparison of the SVP target list of Tao et al. [37] and the list of targets of SVP at the vegetative stage presented here showed that only 15 genes are in common between the two datasets (Additional data file 7, Table S11). This discrepancy might occur for several reasons. First, Tao et al. made use of hybridization to Tiling arrays (ChIP-chip) to identify the genomic regions bound by SVP whereas in the present study these regions were identified by direct sequencing. As described previously, the set of peaks identified by the two technologies can be significantly different [74]. Second, in the ChIP-chip experiments of Tao and collaborators [37]SVP was expressed from the constitutive CaMV35S promoter whereas for the experiments shown here SVP-GFP was expressed from the native SVP promoter. MADS-domain transcription factors (including SVP) are expressed in specific tissues and interact with different partners to bind DNA in a tissue-specific manner [18], so the ectopic expression of SVP in all plant tissues and celltypes, as in $35 \mathrm{~S}:: S V P$ plants, may affect the detection of the binding of this protein to genomic regions in a cell-specific context. Third, Tao et al. [37] identified SVP targets in 9day-old seedlings grown under LDs. In the current study the vegetative tissue was harvested from SVP::SVP-GFP svp- 41 plants grown for 2 weeks under SDs (see Material and Methods). SVP interacting proteins might be expressed differently under these two conditions and therefore affect the capacity and/or selectivity of SVP to bind certain genomic regions.

Previously SVP was shown to delay flowering by directly repressing transcription of $F T$ and $S O C 1$, and reducing the mRNA level of the $F T$ paralogue TSF $[6,18,19]$. Here, direct binding to TSF was not detected suggesting SVP might repress its transcription indirectly. FT and TSF are components of the photoperiodic flowering pathway, while SOC1 is activated by FT in the SAM and acts as a point of convergence of other pathways [75-77]. Analysis of the flowering-time genes present in the high confidence list of SVP targets in vegetative tissue detected other genes acting in the photoperiodic flowering pathway or in the circadian clock that acts upstream of it. Notably, GI and PRR7 are targets of SVP and both are involved in the photoperiodic induction of flowering and circadian clock regulation [78-80]. Both genes are positive regulators of $C O$, which in turn activates $F T$ transcription under long photoperiods. Also the increase in SVP protein accumulation in the lhy ccal double mutant in continuous light, points to a link between SVP regulation and light/clock signaling [55].

The ChIP-seq data suggest that SVP likely also affects flowering by other mechanisms. The $F T$ gene is a target for PRC2 and carries the chromatin mark H3K27me3 $[81,82]$. Therefore the regulation of PRC2 components by SVP may have an indirect effect on $F T$ expression. 
Mutations in components of PRC2, such as CLF that was also identified as a SVP target, cause ectopic expression of MADS-domain proteins that can then promote earlier flowering by mechanisms that remain unclear [83]. Furthermore, PRC2 and other chromatin-related targets of SVP reduce the expression of $F L C$ [84], which encodes another MADS-domain protein that is a strong repressor of flowering and physically interacts with SVP $[18,55,85]$. This complex of FLC and SVP also binds directly to SVP, as discussed later, likely leading to repression of $S V P$ transcription. Thus SVP appears to influence flowering time through several pathways that include chromatin regulation and feedback regulation on its own expression, as well as direct binding to genes encoding components of the circadian clock, photoperiodic flowering pathway and floral integrators.

\section{SVP binds to genes involved in hormonal pathways}

Our ChIP-seq data revealed numerous putative direct targets of SVP involved in hormonal pathways. SVP binds to genes involved in auxin, GA, cytokinin, and jasmonate homeostasis (Additional data file 3, Table S4). One of these direct targets is STIP, a gene involved in the maintenance of the pluripotency and proliferation of meristematic tissue in Arabidopsis [86]. Overexpression of STIP was shown to partially restore the SAM of the cytokinin insensitive ahk2-2 ahk3-3 cre1-12 triple mutants, indicating that STIP acts downstream of CKs in the establishment of the SAM during early seedling development [49]. Several studies detected a role for cytokinins in the promotion of the floral transition [87]. For instance, the mutant altered meristem program 1 (amp1) contains elevated levels of cytokinins and flowers earlier than wild-type plants [88]. Interestingly, the amp1 mutant rescues the late-flowering phenotype of the $g i$ mutant, demonstrating that CK is implicated in the LD pathway downstream of GI [50]. Our qRT-PCR experiments showed that STIP mRNA is induced in svp-41 and in $f t-10$ tsf-1 svp-41 (Figure 6a, b). This result indicates that SVP represses STIP independently or downstream of the two major photoperiod outputs FT and TSF. In addition, the induction of STIP in $s v p-41$ correlates with increased mRNA expression of several cytokinin response genes, belonging to the type-A ARRs and CRFs transcription factor families (Figure 6c), in agreement with the proposed role of STIP in the CK signaling pathway [49]. Moreover, a significant number of genes deregulated in $s v p-41$ were also found to be differentially expressed in response to BA (Figure 6d). These results suggest that in the $s v p-41$ mutant the up-regulation of STIP leads to the activation of the CK signaling pathway.

Additional targets of SVP encode hormonal receptors such as COI1 that may also explain changes in gene expression of signaling components of jasmonate (JAZs genes). Furthermore the auxin responsive genes SAURs increase in expression in $s v p-41$ mutants, and these changes may be caused by altered auxin signaling, as SVP binds directly to genes related to auxin transport, such as BIG [51]. These effects suggest that the developmental role of SVP is likely to involve complex regulation of hormonal signaling pathways.

\section{Common targets of the dimerizing MADS-box factors FLC and SVP}

MADS-box factors form multimeric complexes that are proposed to be important in determining their DNA binding specificity and thereby their function $[15,89]$. SVP interacts with FLC and they are proposed to repress flowering as part of a complex that binds to the SOC1 and $F T$ genes $[6,18,55,77]$. To determine how extensive the overlap in target genes between FLC and SVP is, we compared the vegetative SVP ChIP-seq dataset with the one recently published for FLC [32]. The 112 genes in common between FLC and SVP high confidence targets included CYTOKININ RESPONSE 1 (CRE1/CHASE), supporting a role for both proteins in regulating cytokinin signaling, as discussed above for SVP. However, the ChIP-seq and ChIP-qPCR experiments suggest that SVP and FLC bind to different regions of the gene, with SVP binding in an exon and FLC in the promoter. By contrast, SVP and FLC bound to the same region on the SVP promoter suggesting that the heterodimer composed of SVP and FLC could control SVP expression by means of a feedback loop. Taken together this comparison suggests that FLC and SVP do bind to many genes in similar positions, supporting the idea that they often bind to targets as a heterodimer, however some targets appear to be bound by only one of the proteins, indicating that they also have unique targets. Such a conclusion is consistent with the genetic data, which demonstrated that $s v p \mathrm{flc}$ double mutants flower earlier than either single mutant $[18,55]$.

\section{SVP is linked to meristem function during two phases of development}

Analysis of the subset of SVP targets that is common to vegetative and reproductive development showed an enrichment of genes involved in meristem function. During vegetative development the SAM continuously produces new cells that sustain plant growth by producing leaves and lateral branches, whereas after its formation the FM enlarges in an undifferentiated state until late stage 2, after which floral organ formation is initiated. WUS has a central role in development of both of these stages, participating in the maintenance of the vegetative, inflorescence, and floral meristems [59]. The ChIP-seq analysis showed that SVP binds to regulators of different stages of meristem development and some of these 
converge on the regulation of WUS. The WUS expression domain is restricted to a small group of L3 cells in the center of the meristem by the action of the CLAVATA (CLV) genes [57]. Our data show that SVP binds CLV1 in both vegetative and reproductive tissues and $C L V 2$ in vegetative tissue. Besides the CLAVATA pathway, other genes that restrict WUS expression, for instance HDZIPIII and SPLAYED (SYD) [58,90] are also targets of SVP. In vegetative tissues SVP binds four of the five HDZIPIII genes described in Arabidopsis, $P H B, P H V, R E V$, and $A T H B 8$, and during flower development SVP binds $P H B$. Interestingly, we observed that the patterns of expression of $C L V 1$ and $P H B$ become broader in the SAM of $s v p-41$ mutants compared to Col-0 (Figure 7). These data suggest that SVP influences meristem development by directly binding to genes that act at different levels in the regulatory hierarchy. SVP mRNA abundance in the SAM falls as it undergoes conversion from a vegetative to an inflorescence meristem and this correlates with the meristem becoming more domed and increasing in size $[18,19]$. Reduced activity of SVP in the inflorescence meristem might therefore alter the activity of meristem maintenance pathways to compensate for size differences between the vegetative and inflorescence meristem.

Similarly, floral meristem activity is under control of the MADS-box gene AG, which represses WUS expression after stage 6 of flower development [91]. SVP and AP1 both repress $A G$ expression in the floral meristem, which in turn prevents the repressive activity of $A G$ on WUS. Interestingly, our data show that SVP control CLV1 activity since it binds directly to its locus, in the svp-41 agl24 ap1-12 triple mutant CLV1 is upregulated (Figure 8a) and the induction of SVP-GR result in the downregulation of CLV1; however the pattern of CLV1 expression is retained (Figure $7 \mathrm{k}$ and $\mathrm{l}$ ) suggesting a direct role of SVP in the regulation of CLV1 mRNA quantity, but not in the spatial boundary. Since CLV1 is also involved in repressing WUS activity, the deregulation of $C L V 1$ could be the cause of the downregulation of WUS expression that we detected by in situ (Figure $7 \mathrm{~m}, \mathrm{n})$. Together these data show that SVP and AP1 secure WUS expression in the floral meristem via two pathways: the direct repression of $A G$ and through direct repression of CLV1. This hypothesis is further strengthened by the observation that in the svp-41 agl24. ap1-12 triple mutant a reduction in floral organ number was observed [25], which is probably due to a decrease in meristem size resulting from increased CLV1 activity. Indeed the analysis of floral meristem size that we performed in this study revealed that in the triple mutant the FMs are smaller compared to the wild-type (Figure $7 \mathrm{q}$ and Table 1) indicating a direct correlation between SVP action and different WUS regulatory pathways.

\section{Common targets of AP1 and SVP}

SVP together with AGL24 and AP1 controls floral meristem identity and these proteins are important to prevent early expression of floral homeotic genes, such as AP3, PI, SEP3, and $A G$ in the floral meristem [20]. This repression of floral organ identity genes involves recruitment of the LUG-SEU repressor complex by the AP1-SVP heterodimer [25]. As soon as the sepal primordia start to differentiate from the FM SVP expression disappears, probably due to interaction between AP1 and SEP3, as the latter starts to be expressed during late stage 2 of flower development [92]. Comparison of the gene lists obtained by ChIP-seq experiments for SVP and AP1 [31] identified a significant number of common target genes. Since SVP is strictly expressed in the floral meristem (stages 1 and 2 of flower development), many of these common targets are likely regulated during FM formation rather than specification of floral organ identity. Notably among these common targets transcription factors are enriched. These transcription factors include those involved in meristem maintenance and development. PHB, KAN1, and ARF3 are all bound by both SVP and AP1 and are upregulated in svp-41 agl24 ap1-12 inflorescences and the induction of SVP-GR result in the downregulation of $P H B, K A N 1$, and $A R F 3$ suggesting that SVP modulate their activity. $P H B$, $K A N 1$, and $A R F 3$ are involved in the regulation of meristem development and floral organ formation [58,93-95]. Interestingly the activity of ARFs proteins is controlled by convergent auxin flow that is controlled by PIN proteins and SVP and AP1 bound the genomic region of PIN1, which is expressed in the IM as well as in the FM. Indeed the expression level of PIN1 is repressed by SVP. Taken together, these data suggest that there are interactions between the different regulatory networks that control FM formation and differentiation.

Analysis of the SEP3 ChIP-seq dataset revealed that CLV1, PHB, KAN1, and ARF3 are also bound by SEP3, which also interacts with AP1 [15]. The expression profiles of SVP and SEP3 are mutually exclusive, suggesting a different modulation of the expression of the same target genes by SVP and SEP3 during floral meristem specification and floral meristem differentiation.

\section{SVP targets are enriched in post-transcriptional and post- translational regulators}

Multiple layers of regulation of gene expression play important roles in plant development. Post-transcriptional regulation can enhance and extend the effects of transcriptional regulation. The observation that SVP targets are enriched in genes encoding post-transcriptional and post-translational regulators indicates that SVP may affect gene expression not only by directly binding to target genes and modulating their transcription, but also by indirectly influencing post-transcriptional regulation. 
Protein ubiquitination influences the stability and localization of proteins, resulting in the modulation of their biological functions. Defects in ubiquitination pathways can result in abnormal floral organ identity as suggested by the functional analyses of the DCAF1 and CYP71 genes, which are part of Cullin-RING ubiquitin ligase complexes $[67,96]$.

SVP binds to a large number of DCAF encoding genes in FMs suggesting that SVP could be involved in the control of both proteasome and epigenetically mediated regulation of floral processes (Additional data file 1, Table S9). Several SVP targets are linked to chromatinmediated regulation, such as two uncharacterized WD40 proteins containing Bromodomains, known to bind acetylated lysine residues in histones [97]. Thus SVP likely controls developmental processes by regulating gene expression directly through transcriptional regulation and indirectly by modulating transcription of genes encoding post-transcriptional and post-translational regulators.

It was recently reported that the WDR protein WDR55 is a putative DCAF and may function in a CUL4 DDB1 ${ }^{\text {WDR55 }}$ E3 ligase complex [70]. Interestingly we discovered that WDR55 is a target of SVP, which bound its genomic locus in inflorescence tissues. Moreover WDR55 results downregulated in svp-41 agl24 and svp-41 agl24 ap1-12 compared to the wild-type inflorescences indicating that SVP acts as a direct activator of WDR55 expression in the floral meristem.

\section{The role of WDR55 in floral organ ontogenesis}

The analyses of the mutant $w d r-55-2$ showed variable phenotype in flower development such as reduced number of organs, asymmetric and reduced sepal and petal size, and occasionally chimeric organs such as petaloid stamens and carpelloid stamen or sepals. In-situ hybridization analysis revealed that $A G$ was misexpressed in the $w d r 55-2$ flower. In wild-type, $A G$ expression is always restricted to the two inner whorls (whorls 3 and 4). In homozygous $w d r 55-2$ mutant flowers $A G$ expression is detectable earlier than in wild-type and in all floral whorls. This strongly suggests that WDR55 is involved in both spatial and temporal regulation of $A G$. The SVP-AP1 heterodimer is thought to recruit LUG-SEU and regulate $A G$ expression in early stages of flower development [25]. We tested if WDR55 could bind any of these proteins but were not able to show any interaction.

Taken together the overall data indicate that SVP repress $A G$ expression through two different pathways, the first is via the interaction with the co-repressor complex containing LUG-SEU and the dimer SVP-AP1 [25] and the second by SVP controlling the expression level of WDR55. The floral phenotype of the $w d r 55-2$ mutant is variable and did not result in the deregulation of $A G$ in all the flowers, this suggests that SVP in the $w d r 55-2$ background is, although less efficient, still able to repress $A G$ directly probably via the LUG-SEU pathway.

\section{Conclusions}

In summary, our data indicate that the SVP genomewide binding profiles during two distinct developmental stages show a significant overlap and that this subset of genes includes a wider set of important regulators of plant development than was previously realized. However, there is also a large group of SVP target genes that are not bound at both stages, clearly reflecting distinct functions during vegetative and reproductive phases. The specificity of SVP binding to DNA is probably influenced by interaction with different MADS-domain partners, such as FLC and AP1. A related observation was made for the Drosophila MADS domain protein MEF2 that is expressed widely during development, but has specific targets at different stages dependent on the presence of interacting transcription factors [98]. The presented data provide new insights into the enormous diversity of pathways that are regulated by SVP and forms a basis for detailed analysis of the roles of SVP in regulating specific genes and pathways in combination with different interacting proteins.

\section{Materials and methods}

\section{Plant material and growth conditions}

For ChIP and microarray analysis of vegetative phase, SVP::SVP-GFP, svp-41 single mutant (for plasmid construction see [20]) and wild-type seedlings were grown 14 days under short-day (SD) conditions ( $8 \mathrm{~h} \mathrm{light/16} \mathrm{h}$ dark) at $22^{\circ} \mathrm{C}$. For ChIP and microarray analysis of the reproductive phase, SVP::SVP-GFP svp-41, triple mutant svp-41 agl24-2 ap1-12 and wild-type plants were grown under long-day (LD) conditions (LD; $16 \mathrm{~h}$ light $/ 8 \mathrm{~h}$ dark) at $22^{\circ} \mathrm{C}$. For the GR induction study the triple mutant svp-41 agl24-2 ap1-10 was used [24]. All the plants were from the same Columbia ecotype. The SVP:: SVP-GFP svp-41 transgenic line and triple mutant svp41 agl24-2 ap 1-12 have been previously described $[20,25]$. ft-10 tsf- 1 svp-41 and $f t-10$ tsf- 1 were described previously in Jang et al. [19]. The $w d r 55-2$ (WiscDsLox430F06) line is in the Col-0 ecotype and is a TDNA insertion mutant obtained from the Nottingham Arabidopsis Stock Centre [99]. Seeds were surface sterilized using EtOH, bleach and Tween 20 before germinated on MS media [100] supplemented with $2 \%$ sucrose (MS-2) and glufosinate-ammonium for BASTA selection of $w d r 55-2$ plants. All seeds were stratified on MS-2 plates at $4^{\circ} \mathrm{C}$ O.N. before being transferred to $18^{\circ} \mathrm{C}$ for 
about 12 days until germination. The seedlings were eventually transferred to soil and grown at $18^{\circ} \mathrm{C}$ under LD conditions (16 h).

\section{ChIP assays}

For ChIP experiments, the commercial antibody GFP: Living Colors_full-length A.v. polyclonal antibody was used (Clontech [101]). Chromatin was prepared from inflorescences (2 weeks after bolting) and from 14-dayold seedlings of $s v p$, grown under SD conditions. Wildtype plants (inflorescences and seedlings) were used as negative controls. ChIP assays were performed as previously described by [20] and in Additional data file 1, Methods S1 with a minor modification in the sonication step. DNA samples were sonicated six times $30 \mathrm{~s}$ each with amplitude 30 to 40, with intervals of $1 \mathrm{~min}$ (100500 bp range fragments obtained).

We used as a positive control for the ChIP in the reproductive phase a region of the $A G$ second intron (AG.V) that previously has been demonstrated to bind SVP-GFP [20]. For the vegetative phase we used regions in $F T$ bound by SVP [18] (Additional data file 1, Figure S1). Enrichment fold to evaluate the quality of each ChIP sample was tested by qRT-PCR as described in Additional data file 1, Methods S2, all the primers used for ChIP-qPCR are in Additional data file 1, Table S12).

\section{Sample preparation for ChIP-seq Illumina/Solexa sequencing}

Two independent ChIP experiments (enrichment fold controlled by real-time PCR) were used for vegetative and reproductive ChIP-seq assays, respectively. We used one ChIP DNA sample for each library preparation and these were run on the Genome Analyzer. The DNA quantification of immunoprecipitated DNA was performed with the Quant-iT dsDNA HS Assay Kit (Invitrogen). Libraries for Solexa sequencing were prepared following the Illumina kit protocol, with some modifications. The first step 'Perform End Repair' was repeated twice, adding fresh enzymes and incubating $1 \mathrm{~h}$ longer than indicated by the protocol. Two units of undiluted Klenow enzyme was used. The incubation time of the step 'Ligate adapters to DNA fragments' was prolonged to $1 \mathrm{~h}$ instead of $15 \mathrm{~min}$. Each library was validated quantifying the DNA with Quant-iT dsDNA HS Assay Kit (Invitrogen).

\section{Read mapping and identification of enriched regions}

Sequence reads were mapped to the unmasked Arabidopsis genome (TAIR8 build) using the Seqmap tool [102], allowing at most two mismatches at any position. Trimming unmapped reads at the $5^{\prime}$ or 3 ' end led to marginal improvements in the number of reads mapped, and this step was therefore skipped. Reads belonging to duplicate experiments in each of the three conditions were pooled together. Only reads mapping to a unique position on the genome were considered for further analysis. This resulted in about 3 million uniquely mapped reads for the two inflorescences experiments, 5 million for seedlings experiments, and 6 million for control experiments. In each experiment, uniquely mapped reads were extended by 300 bps along the 5 ' $->3$ ' direction. This resulted in a base pair by base pair coverage map of the genome, that is, giving for each base pair the number of extended sequence reads that contained it. Only base pairs covered by reads mapping on both strands were considered valid for further analysis. Enrichment was then calculated in each valid base pair by comparing, for each IP experiment, the coverage in the experiment to the coverage in the control used as expected value, and computing an enrichment $P$ value with a negative binomial distribution. In each comparison, the coverage of the two samples was normalized according to the number of reads obtained in each. Enriched regions were then defined as regions consisting of consecutive base pairs characterized by calculated $P$ values $<0.01$ and not interrupted by a gap of 100 or more base pairs that were either non-valid or with a $P$ value $>0.01$. The $P$ value associated with each of these regions was defined as the minimum $P$ value among the base pairs belonging to the region. Regions $<150$ bps were then discarded regardless of the $P$ value. The number of remaining candidate-enriched regions was finally used to compute a Bonferroni corrected $P$ value to be associated to the regions themselves. The overall strategy we followed in our analysis for the identification of enriched regions is highly similar to the one adopted in the SEP3 and AP1 ChIP-Seq experiments [13,31] and in the CSAR peak-finding tool [41], which has been shown to be better suited for ChIP-Seq experiments in Arabidopsis. $P$ values for enrichment were computed by using a negative binomial distribution instead of the Poisson, as the former provides a better fit to count data from ChIP-Seq experiments [103]. Also, we employed a more conservative Bonferroni correction for multiple testing aimed at minimizing the number of false positive predictions.

Starting from regions with corrected $P$ values $<0.01$, potential target genes were then identified by associating with each gene an overall $P$ value given by the product of the $P$ values associated with the single binding regions located in its gene locus, from $3 \mathrm{kbps}$ upstream of the transcription start site to $1 \mathrm{kbp}$ downstream of the transcribed region. Protocols of ChIP, DNA extraction, sequencing preparation, data processing, and all the associated files to this study can be found in the GEO (Gene Expression Omnibus) database (ID: GSE33120). 


\section{Tiling array experiments}

The vegetative tissue samples were obtained from aerial parts of the svp- 41 single mutant and wild-type seedlings grown for 2 weeks under SD conditions $(8 \mathrm{~h}$ light/16 h dark) and harvested at zeitgeber 8 (ZT8). For the reproductive tissue sampling we used wild-type and $s v p-41$ agl24-2 ap 1-12 triple mutant inflorescences grown for 2 weeks under SD conditions and then moved to LD conditions ( $16 \mathrm{~h}$ light $/ 8 \mathrm{~h}$ dark). The inflorescences were collected at 2 weeks after bolting at ZT8. RNA from three independent biological replicates was extracted using the RNA Plant Mini kit, QIAGEN (www1.qiagen.com/) and quantified by NanoDrop; $1 \mu \mathrm{g}$ of total RNA was reverse transcribed into cDNA using an oligo(dT)-T7 primer, and was then converted into cRNA and linearly amplified by T7 in-vitro transcription reaction using the standard Ambion protocol (MessageAmp aRNA Kit, Ambion). cRNA was then reverse transcribed with random primers to dUTP-containing ds cDNA (WT ds cDNA Synthesis Kit, catalog no. 900813; Affymetrix). Fragmentation and labeling was performed with the GeneChip WT doublestranded DNA Terminal Labeling Kit (catalog no. 900812, Affymetrix). After fragmentation, 7.5 ug of dscDNA was hybridized for $16 \mathrm{~h}$ at $45^{\circ} \mathrm{C}$ on GeneChip Arabidopsis Tiling 1.0R Array. GeneChips were washed and stained with Fluidics Script FS450_0001 in the Affymetrix Fluidics Station 450. Then, the GeneChips were scanned using the GeneChip Scanner 3000 7G. Data were processed in R as described in [104]. Probe-level data were pre-processed using the RMA algorithm implemented in the Bioconductor package Affy. Linear models and empirical Bayes methods from the Limma package of Bioconductor were applied to derive a $P$ value, false discovery rate (FDR; P adjusted), and mean of $\log 2$-based ratio across replicates. The data were deposited in the GEO (Gene Expression Omnibus) database (ID: GSE32397).

\section{Gene Ontology analysis}

The Bingo 2.44 plug-in [105] implemented in Cytoscape v2.81 [106] was used to determine and visualize the GO enrichment according to the GOslim categorization. A hypergeometric distribution statistical testing method was applied to determinate the enriched genes and the Benjamini and Hochberg FDR correction was performed in order to limit the number of false positives. The FDR was set up to 0.001 and 0.05 for the ChIP-seq and expression data, respectively. In addition to Bingo 2.44, further GO annotation analysis of the targets of SVP was performed by using TAIR bioinformatics resources [107].

\section{CDNA preparation and qRT-PCR analysis}

Expression analyses in the vegetative phase was performed using the $s v p-41$ single mutant, $35 S:: S V P$ and wild-type seedlings grown for 2 weeks under SD conditions; for the reproductive phase we used wild-type and svp-41 agl24-2 ap1-12 triple mutant inflorescences grown for 2 weeks under SD conditions and then moved to LD conditions. The inflorescences were collected at 2 weeks after bolting.

Total RNA from three biological replicates was extracted with the $\mathrm{LiCl}$ method, and its integrity was checked on agarose gels. The samples were treated with DNase (TURBO DNA-free; Ambion [108]) and reverse transcribed according to the ImProm-II_ Reverse Transcription System (Promega [109]) instructions. Sequence primers for RT-PCR amplification are listed in Additional data file 1, Table S13. Ten-fold dilutions of cDNA were tested in RT-PCR and qRT-PCR experiments using reference genes.

Enrichment folds were detected using a SYBR Green assay (Bio-Rad [110]). The real-time PCR assay was performed in triplicate using a Bio-Rad C1000 Thermal Cycler optical system or LightCycler480 (ROCHE) thermal cycler. For expression analyses normalized expression was calculated using the delta-delta $\mathrm{Ct}$ method $(D D C(t))$. For ChIP experiments, relative enrichment was calculated as described in Additional data file 1, Methods S2. For the expression analysis ubiquitin, PEX4, and PP2a-F were used as reference genes.

\section{In-situ hybridization}

In-situ hybridization has been performed as described in Additional data file 1, Method S3. The WUS antisense probe has been cloned according to Brambilla et al. [111]. The ARF3 antisense probe has been cloned in the pGEM$\mathrm{T}$ easy using the primers FW-CCCATCTGTATCATCATCACC and REV- CTCTCATTGCATAGATGTCC. The KAN1 antisense probe has been cloned in the pGEM$\mathrm{T}$ easy using the primers FW- AAGACCACTAACAAGCCTGC and REV-CATTTCTCGTGCCAATC TGGTC. The $C L V 1$ antisense probe has been cloned according to Clark et al. [60]. The PHB antisense probe has been cloned in the pGEM-T easy using the primers FW-GGTAGCGATGGTGCAGAGG and REV-CGAACGACCAATTCACGAAC. Sections were observed using a Zeiss Axiophot D1 microscope (Zeiss [112]) equipped with differential interface contrast (DIC) optics. Images were captured on an Axiocam MRc5 camera (Zeiss) using the AXIOVISION program (version 4.4).

\section{Scanning electron microscopy}

SEM has been performed as described in Additional data file 1, Method S4.

\section{Inducible expression experiments}

The $p 35 S:: S V P-G R$ construct was produced as follows: the coding region of $S V P$ was amplified from inflorescence 
cDNA using primers Fw-CGTTGCCATGGCGAGAGAA AAGAT and Rev- ATTGTTCGGATCCCCACCACCATACGG containing NcoI and BamHI sites, respectively, cloned into pGEM-T easy (Promega), digested with NcoI and BamHI and ligated into pBluescript SK (Stratagene) containing a portion of the rat glucocorticoid hormone binding domain (a.a 508-795 [61]) to produce $p S K-S V P$ $G R$. The AG-GR fragment was amplified from the $p S K$ $S V P-G R$ using the primers For and Rev and subcloned into the pTOPO vector (Life Technology). Finally $S V P-G R$ was subcloned into the Gateway destination vector pB2GW7.0 [113] containing the 35S promoter. p35S::SVPGR was transformed in svp-41 agl24-2 ap1-10 background (ap1-10 heterozygous) and the T1 generation was selected for BASTA resistance.

After bolting, inflorescences of 35S::SVP-GR svp-41 agl24-1 ap1-10 plants were treated with a solution containing $10 \mu \mathrm{M}$ dexamethasone (Sigma-Aldrich), 0.01\% $(\mathrm{v} / \mathrm{v})$ ethanol, and $0.015 \%(\mathrm{v} / \mathrm{v})$ Silwet L-77. Mock treatment consist of $0.01 \%(\mathrm{v} / \mathrm{v})$ ethanol, and $0.015 \%(\mathrm{v} / \mathrm{v})$ Silwet L-77.

For each time point, tissue from eight plants was collected. Tissue was removed as close to the surface of the inflorescence as possible to ensure an enrichment of FM cells.

\section{Appendix}

\section{Accession numbers}

Arabidopsis Genome Initiative locus identifiers for the genes mentioned in this article are as follows: AGL24 [TAIR:AT4G24540], STK [TAIR:AT4G09960], AP3 [TAIR:AT3G54340], FLC [TAIR:AT5G10140], SVP [TAIR:AT2G22540], JAZ6 [TAIR:AT1G72450], AGL16 [TAIR:AT3G57230], SOC1 [TAIR:AT2G45660], CLV1 [TAIR:AT1G75820], PIN1 [TAIR:AT1G73590], ARF3/ ETT [TAIR:AT2G33860], KAN1 [TAIR:AT5G16560], $P H B$ [TAIR:AT2G34710], JAZ7 [TAIR:AT2G34600], SADHU [TAIR:AT3G42658], JAZ8 [TAIR:AT1G30135], GA2ox6 [TAIR:AT1G02400], ARR6 [TAIR:AT5G62920], ARR7 [TAIR:AT1G19050], DDF1 [TAIR:AT1G12610], GA2ox2 [TAIR:AT1G30040], miR167 [TAIR:AT1G31173], ACD6 [TAIR:AT4G14400], AP1 [TAIR:AT1G69120], WDR55 [TAIR:AT2G34260], VRN2 [TAIR: AT4G16845], CLF [TAIR: AT2G23380], SWN [TAIR: AT4G02020], GI [TAIR: AT1G22770], FLK [TAIR: AT3G04610], FLD [TAIR: AT3G10390], PRR7 [TAIR: AT5G02810], PHYA [TAIR: AT1G09570], STIP [TAIR: AT2G33880], ARR11 [TAIR: AT1G67710], ARR5 [TAIR: AT3G48100], ARR15 [TAIR: AT1G74890], CRF2 [TAIR: AT4G23750], CRF5 [TAIR: AT2G46310], $P H V$ [TAIR: AT1G30490], REV [TAIR: AT5G60690], ATHB8 [TAIR: AT4G32880], ATBARD1 [TAIR: AT1G04020], KAN2 [TAIR: AT1G32240], LMI1 [TAIR: AT5G03790], DCAF1 [TAIR:
AT4G31160], JAZ5 [TAIR: AT1G17380], JAZ10 [TAIR: AT5G13220], JAZ1 [TAIR: AT1G19180]

\section{Additional material}

\begin{abstract}
Additional data file 1: contains: Figure S1: Analysis of chromatin sample used for ChIP-seq experiments. Figure S2: qRT-PCR validation of differentially expressed genes between Col-0 and svp-41 plants at the vegetative phase. Figure S3: GO enrichment analysis of differentially expressed genes between Col-0 and svp-41 plants at the vegetative stage. Figure S4: Venn diagram containing the overlapping set of putative targets between SVP and FLC and SVP, AP1, and SEP3. Figure S5: Biologically active SVP-GR fusion. Figure S6: Flower organs of in wdr55-2 -/- mutants show reduced size and asymmetric positioning. Figure S7: In-situ hybridization of wild-type and wdr55-2 inflorescence using AP3 and PI probes. Figure S8: Flower morphology of wdr55-2 ag-1 mutant. Table S1: Summary of sequencing and mapping. Table S3: List of putative targets of SVP related to flowering time. Table S6: List of genes differentially expressed in svp-41 compare to Col-0 and related to auxin, cytokinin, or jasmonate homeostasis. Table S9: List of WDxR motif containing proteins found in SVP DNA binding screen. Table S10: Flower organ count from wdr55-2 -/- mutants. Table S12: Primer pairs used for ChIP-qPCR assays. Table S13: Primer pairs used for the GRT-PCR expression analysis. Methods S1: ChIP protocol. Methods S2: qRT-PCR. Methods S3: In-situ hybridization. Methods S4: Scanning electron microscopy.

Additional data file 2: contains Table S2: High confidence targets of SVP in vegetative and reproductive tissues; list of the targets of SVP bound in both vegetative and reproductive tissues; lists of binding regions of SVP in vegetative and reproductive tissues.
\end{abstract}

Additional data file 3: contains Table S4: Lists of putative SVP targets with annotated functions in: meristem development in vegetative and reproductive tissues; response to hormonal stimuli such as auxin, cytokinin, ethylene, abscisic acid, jasmonate, and gibberellins in vegetative tissue

Additional data file 4: contains Table S5: Tiling array expression data obtained using RNA extracted from: wild-type Col-0 and svp-41 plants at the vegetative stage, inflorescences of wild-type Col-0 and svp-41 ag/24 ap 1-12 and overlap between tiling array and ChIP-seq data.

Additional data file 5: contains Table S7: Lists of differentially expressed genes in svp-41 mutant and the available expression-profiling data of seedlings treated with the CK benzyladenine (BA).

Additional data file 6: contains Table S8: putative targets for both SVP and AP1 and putative targets for both SVP and SEP3.

Additional data file 7: contains Table S8: Comparison of the SVP target list of Tao et al. [37] and the list of high confidence targets of SVP in vegetative tissue presented in this study.

\section{Abbreviations}

CArG-boxes: MADS-domain factors binding consensus; ChIP-qPCR: chromatin immunoprecipitation followed by quantitative real-time polymerase chain reaction; ChIP-seq: chromatin immunoprecipitation combined with high throughput DNA sequencing; ChIP: chromatin immunoprecipitation; CK: cytokinin; CSAR: ChIP-seq analysis in R; FM: floral meristem; GA: gibberellin; GEO: Gene Expression Omnibus; GO: Gene Ontology; GR: glucocorticoid receptor; IM: inflorescence meristem; qRT-PCR: quantitative real-time polymerase chain reaction; SAM: shoot apical meristem; Y2H: Yeast-2-Hybrid

\section{Authors' contributions}

VG did the ChIP experiments, biological analysis, and writing; FA did the transcriptome analysis, biological analysis, and writing; AS did the ChIP-seq and writing; RFG, SS, JLM, ST, and KNB performed the biological analysis; FZ and GMP were responsible for bioinformatics; GP did the bioinformatics and writing; LC conducted the research design; PEG, GC, and MMK did the 
research design and writing. All authors read and approved the final manuscript.

\section{Acknowledgements}

We thank V. Grandi, Marco Passaro, and Virginia Borrelli (Università degli Studi di Milano), K. Kaufmann (Wageningen University and Research Center, Wageningen) for technical support. Bruno Huettel (Max Planck Institute, Cologne) for the tiling array experiments. The SEM analysis was conducted at Centro Interdipartimentale di Microscopia Avanzata (CIMA) with the help of Dr. E. Caporali. We thank also Peter Huijser (Max Planck Institute, Cologne) for providing 355::SVP seeds. The post-doctoral and PhD fellowships for VG, SS, and FZ are funded by the Università degli Studi di Milano. FA is the recipient of a Marie Curie Intra-European Fellowship for Career Development (PIEF-GA-2009-251839). KNB and PEG were supported by grants from the Norwegian Research Council to PEG (grants 183190/S10 and 214052/F20). JLM is the recipient of a Humboldt Research Fellowship for Postdoctoral Researchers. GC received a grant from ERA-NET PG within the BLOOMNET project. This work was supported by the FLOWER POWER project (ID AGRO11 and Ref. $n^{\circ}$ 16976) of the Lombardia region, Italy.

\section{Author details}

'Department of Bioscience, Università degli Studi di Milano, Via Celoria 26, 20133 Milan, Italy. ${ }^{2}$ Max Planck Institute for Plant Breeding Research, D-50829 Cologne, Germany. ${ }^{3}$ Department of Biosciences, University of Oslo, N-0316 Oslo, Norway. ${ }^{4}$ Consiglio Nazionale delle Ricerche Istituto di Biofisica, 20133 Milan, Italy.

Received: 24 March 2013 Revised: 24 April 2013

Accepted: 11 June 2013 Published: 11 June 2013

\section{References}

1. Irish VF: Patterning the flower. Dev Biol 1999, 209:211-220.

2. Jack T: Molecular and genetic mechanisms of floral control. Plant Cell 2004, Suppl 1: S1-17.

3. Ma H: To be, or not to be, a flower-control of floral meristem identity. Trends Genet 1998, 14:26-32.

4. Melzer S, Lens F, Gennen J, Vanneste S, Rohde A, Beeckman T: Floweringtime genes modulate meristem determinacy and growth form in Arabidopsis thaliana. Nat Genet 2008, 40:1489-1492.

5. Andrés F, Coupland G: The genetic basis of flowering responses to seasonal cues. Nat Rev Genet 2012, 13:627-639.

6. Lee JH, Yoo SJ, Park SH, Hwang I, Lee JS, Ahn JH: Role of SVP in the control of flowering time by ambient temperature in Arabidopsis. Genes Dev 2007, 21:397-402.

7. Mouradov A, Cremer F, Coupland G: Control of flowering time: interacting pathways as a basis for diversity. Plant Cell 2002, Suppl 1: S111-S130.

8. Hartmann U, Hohmann S, Nettesheim K, Wisman E, Saedler H, Huijser P: Molecular cloning of SVP: a negative regulator of the floral transition in Arabidopsis. Plant J 2000, 21:351-360.

9. Parenicová L, de Folter S, Kieffer M, Horner DS, Favalli C, Busscher J, Cook HE, Ingram RM, Kater MM, Davies B, Angenent GC, Colombo L: Molecular and phylogenetic analyses of the complete MADS-box transcription factor family in Arabidopsis: new openings to the MADS world. Plant Cell 2003, 15:1538-1551.

10. de Folter S, Angenent GC: Trans meets cis in MADS science. Trends Plant Sci 2006, 11:224-231.

11. Colombo M, Masiero S, Vanzulli S, Lardelli P, Kater MM, Colombo L: AGL23, a type I MADS-box gene that controls female gametophyte and embryo development in Arabidopsis. Plant J 2008, 6:1037-1048.

12. Masiero S, Colombo L, Grini PE, Schnittger A, Kater MM: The emerging importance of type I MADS box transcription factors for plant reproduction. Plant Cell 2011, 23:865-872.

13. Kaufmann K, Muino JM, Jauregui R, Airoldi CA, Smaczniak C, Krajewski P, Angenent GC: Target genes of the MADS transcription factor SEPALLATA3: integration of developmental and hormonal pathways in the Arabidopsis flower. PLOS Biol 2009, 7:e1000090.

14. Theissen G, Saedler H: Plant biology. Floral quartets. Nature 2001, 409:469-471.

15. de Folter S, Immink RG, Kieffer M, Parenicová L, Henz SR, Weigel D, Busscher M, Kooiker M, Colombo L, Kater MM, Davies B, Angenent GC:
Comprehensive interaction map of the Arabidopsis MADS Box transcription factors. Plant Cell 2005, 17:1424-1433.

16. Messenguy F, Dubois E: Role of MADS box proteins and their cofactors in combinatorial control of gene expression and cell development. Gene 2003, 316:1-21.

17. Simonini S, Roig-Villanova I, Gregis V, Colombo B, Colombo L, Kater MM: Basic pentacysteine proteins mediate MADS domain complex binding to the DNA for tissue-specific expression of target genes in Arabidopsis. Plant Cell 2012, 24:4163-4172.

18. Li D, Liu C, Shen L, Wu Y, Chen H, Robertson M, Helliwell CA, Ito T, Meyerowitz $\mathrm{E}, \mathrm{Yu} \mathrm{H}$ : A repressor complex governs the integration of flowering signals in Arabidopsis. Dev Cell 2008, 15:110-120.

19. Jang S, Torti S, Coupland G: Genetic and spatial interactions between FT, TSF and SVP during the early stages of floral induction in Arabidopsis. Plant J 2009, 60:614-625.

20. Gregis $V$, Sessa A, Dorca-Fornell C, Kater MM: The Arabidopsis floral meristem identity genes AP1, AGL24 and SVP directly repress class B and C floral homeotic genes. Plant J 2009, 60:626-637.

21. $Y u H, X u Y$, Tan EL, Kumar PP: AGAMOUS-LIKE 24, a dosage-dependent mediator of the flowering signals. Proc Natl Acad Sci USA 2002, 99:16336-16341

22. Michaels SD, Ditta G, Gustafson-Brown C, Pelaz S, Yanofsky M, Amasino RM: AGL24 acts as a promoter of flowering in Arabidopsis and is positively regulated by vernalization. Plant J 2003, 33:867-874

23. Liu C, Chen H, Er HL, Soo HM, Kumar PP, Han JH, Liou YC, Yu H: Direct interaction of AGL24 and SOC1 integrates flowering signals in Arabidopsis. Development 2008, 135:1481-1491.

24. Gregis V, Sessa A, Colombo L, Kater MM: AGAMOUS-LIKE24 and SHORT VEGETATIVE PHASE determine floral meristem identity in Arabidopsis. Plant J 2008, 56:891-902.

25. Gregis V, Sessa A, Colombo L, Kater MM: AGL24, SHORT VEGETATIVE PHASE, and APETALA1 redundantly control AGAMOUS during early stages of flower development in Arabidopsis. Plant Cell 2006, 18:1373-1382.

26. Kempin SA, Savidge B, Yanofsky MF: Molecular basis of the cauliflower phenotype in Arabidopsis. Science 1995, 267:522-525.

27. Johnson D S, Mortazavi A, Myers RM, Wold B: Genome-wide mapping of in vivo protein-DNA interactions. Science 2007, 316:1497-1502.

28. Robertson AG, Bilenky M, Tam A, Zhao Y, Zeng T, Thiessen N, Cezard T, Fejes AP, Wederell ED, Cullum R, Euskirchen G, Krzywinski M, Birol I, Snyder M, Hoodless PA, Hirst M, Marra MA, Jones SJ: Genome-wide relationship between histone $\mathrm{H} 3$ lysine 4 mono- and tri-methylation and transcription factor binding. Genome Res 2008, 18:1906-1917.

29. Nielsen R, Pedersen TA, Hagenbeek D, Moulos P, Siersbaek R, Megens E, Denissov S, Børgesen M, Francoijs KJ, Mandrup S, Stunnenberg HG: Genome-wide profiling of PPARgamma:RXR and RNA polymerase II occupancy reveals temporal activation of distinct metabolic pathways and changes in RXR dimer composition during adipogenesis. Genes Dev 2008, 22:2953-2967.

30. Wederell ED, Bilenky M, Cullum R, Thiessen N, Dagpinar M, Delaney A, Varhol R, Zhao Y, Zeng T, Bernier B, Ingham M, Hirst M, Robertson G, Marra MA, Jones S, Hoodless PA: Global analysis of in vivo Foxa2-binding sites in mouse adult liver using massively parallel sequencing. Nucleic Acids Res 2008, 36:4549-4564.

31. Kaufmann K, Wellmer F, Muino JM, Ferrier T, Wuest SE, Kumar V, SerranoMislata A, Madueño F, Krajewski P, Meyerowitz EM, Angenent GC, Riechmann $\mathrm{J}$ : Orchestration of floral initiation by APETALA1. Science 2010, 328:85-89.

32. Deng W, Ying H, Helliwell CA, Taylor JM, Peacock WJ, Dennis ES: FLOWERING LOCUS C (FLC) regulates development pathways throughout the life cycle of Arabidopsis. Proc Natl Acad Sci USA 2011, 108:6680-6685.

33. Immink RG, Posé $D$, Ferrario $S$, Ott F, Kaufmann $K$, Valentim FL, de Folter $S$, van der Wal F, van Dijk AD, Schmid M, Angenent GC: Characterization of SOC1's central role in flowering by the identification of its upstream and downstream regulators. Plant Physiol 2012, 160:433-449.

34. Yant L, Mathieu J, Dinh TT, Ott F, Lanz C, Wollmann H, Chen X, Schmid M: Orchestration of the floral transition and floral development in Arabidopsis by the bifunctional transcription factor APETALA2. Plant Cell 2010, 22:2156-2170. 
35. Zheng Y, Ren N, Wang H, Stromberg AJ, Perry SE: Global identification of targets of the Arabidopsis MADS domain protein AGAMOUS-Like15. Plant Cell 2009, 21:2563-2577.

36. Winter CM, Austin RS, Blanvillain-Baufume S, Reback MA, Monniaux M, Wu MF, Sang Y, Yamaguchi A, Yamaguchi N, Parker JE, Parcy F, Jensen ST, Li H, Wagner D: LEAFY target genes reveal floral regulatory logic, cis motifs, and a link to biotic stimulus response. Dev Cell 2011, 20:430-443.

37. Tao Z, Shen L, Liu C, Liu L, Yan Y, Yu H: Genome-wide identification of SOC1 and SVP targets during the floral transition in Arabidopsis. Plant $J$ 2012, 70:549-561.

38. Chalfie M, Tu Y, Euskirchen G, Ward WW, Prasher DC: Green fluorescent protein as a marker for gene expression. Science 1994, 263:802-805.

39. Liu C, Zhou J, Bracha-Drori K, Yalovsky S, Ito T, Yu H: Specification of Arabidopsis floral meristem identity by repression of flowering time genes. Development 2007, 134:1901-1910.

40. Smyth DR, Bowman JL, Meyerowitz EM: Early flower development in Arabidopsis. Plant Cell 1990, 2:755-767.

41. Muiño JM, Kaufmann K, van Ham RC, Angenent GC, Krajewski P: ChIP-seq Analysis in R (CSAR): An R package for the statistical detection of protein-bound genomic regions. Plant Methods 2011, 7:11.

42. Liljegren SJ, Gustafson-Brown C, Pinyopich A, Ditta GS, Yanofsky MF: Interactions among APETALA1, LEAFY, and TERMINAL FLOWER1 specify meristem fate. Plant Cell 1999, 11:1007-1018.

43. Shore $P$, Sharrocks AD: The MADS-box family of transcription factors. Eur $J$ Biochem 1995, 229:1-13.

44. Meyerowitz EM: DNA-binding properties of Arabidopsis MADS domain homeotic proteins APETALA1, APETALA3, PISTILLATA and AGAMOUS. Nucleic Acids Res 1996, 24:3134-3141.

45. Causier BE, Davies B, Sharrocks AD: DNA binding and dimerisation determinants of Antirrhinum majus MADS-box transcription factors. Nucleic Acids Res 1998, 26:5277-5287.

46. Tang W, Perry SE: Binding site selection for the plant MADS domain protein AGL15: an in vitro and in vivo study. J Biol Chem 2003, 278:28154-28159.

47. Pavesi G, Mereghetti P, Mauri G, Pesole G: Weeder Web: discovery of transcription factor binding sites in a set of sequences from coregulated genes. Nucleic Acids Res 2004, 34(Web Server):W566-570.

48. Liu C, Xi W, Shen L, Tan C, Yu H: Regulation of floral patterning by flowering time genes. Dev Cell 2009, 16:711-722.

49. Skylar A, Hong F, Chory J, Weigel D, Wu X: STIMPY mediates cytokinin signaling during shoot meristem establishment in Arabidopsis seedlings. Development 2010, 137:541-549.

50. Bernier G, Perilleux C: A physiological overview of the genetics of flowering time control. Plant Biotechnol 2005, 3:3-16.

51. Gil P, Dewey E, Friml J, Zhao Y, Snowden KC, Putterill J, Palme K, Estelle M, Chory J: BIG: a calossin-like protein required for polar auxin transport in Arabidopsis. Genes Dev 2001, 15:1985-1997.

52. Yamaguchi N, Suzuki M, Fukaki H, Morita-Terao M, Tasaka M, Komeda Y: CRM1/BIG-mediated auxin action regulates Arabidopsis inflorescence development. Plant Cell Physiol 2007, 48:1275-1290.

53. Yan J, Zhang C, Gu M, Bai Z, Zhang W, Qi T, Cheng Z, Peng W, Luo H, Nan F, Wang Z, Xie D: The Arabidopsis CORONATINE INSENSITIVE1 protein is a jasmonate receptor. Plant Cell 2009, 21:2220-2236.

54. Sheard LB, Tan X, Mao H, Withers J, Ben-Nissan G, Hinds TR, Kobayashi Y, Hsu FF, Sharon M, Browse J, He SY, Rizo J, Howe GA, Zheng N: Jasmonate perception by inositol-phosphate-potentiated COI1-JAZ co-receptor. Nature 2010, 468:400-405.

55. Fujiwara S, Oda A, Yoshida R, Niinuma K, Miyata K, Tomozoe Y, Tajima T, Nakagawa M, Hayashi K, Coupland G, Mizoguchi T: Circadian clock proteins LHY and CCA1 regulate SVP protein accumulation to control flowering in Arabidopsis. Plant Cell 2008, 20:2960-2971.

56. Kurihara Y, Matsui A, Hanada K, Kawashima M, Ishida J, Morosawa T, Tanaka M, Kaminuma E, Mochizuki Y, Matsushima A, Toyoda T, Shinozaki K, Seki M: Genome-wide suppression of aberrant mRNA-like noncoding RNAs by NMD in Arabidopsis. Proc Natl Acad Sci USA 2009, 106:2453-2458.

57. Schoof H, Lenhard M, Haecker A, Mayer KF, Jurgens G, Laux T: The stem cell population of Arabidopsis shoot meristems in maintained by a regulatory loop between the CLAVATA and WUSCHEL genes. Cell 2000, 100:635-644

58. Prigge MJ, Otsuga D, Alonso JM, Ecker JR, Drews GN, Clark SE: Class III homeodomain-leucine zipper gene family members have overlapping, antagonistic, and distinct roles in Arabidopsis development. Plant Cell 2005, 17:61-76.

59. Sablowski R: Flowering and determinacy in Arabidopsis. J Exp Bot 2007, 58:899-907.

60. Clark SE, Williams RW, Meyerowitz EM: The CLAVATA1 gene encodes a putative receptor kinase that controls shoot and floral meristem size in Arabidopsis. Cell 1997, 89:575-585.

61. Gómez-Mena C, de Folter S, Costa MM, Angenent GC, Sablowski R: Transcriptional program controlled by the floral homeotic gene AGAMOUS during early organogenesis. Development 2005, 132:429-438.

62. Schwechheimer C, Calderon Villalobos LI: Cullin-containing E3 ubiquitin ligases in plant development. Curr Opin Plant Biol 2004, 7:677-686.

63. Dumbliauskas E, Lechner E, Jaciubek M, Berr A, Pazhouhandeh M, Alioua M, Cognat V, Brukhin V, Koncz C, Grossniklaus U, Molinier J, Genschik P: The Arabidopsis CUL4-DDB1 complex interacts with MSI1 and is required to maintain MEDEA parental imprinting. EMBO J 2011, 30:731-743.

64. He YJ, McCall CM, Hu J, Zeng Y, Xiong Y: DDB1 functions as a linker to recruit receptor WD40 proteins to CUL4-ROC1 ubiquitin ligases. Genes Dev 2006, 20:2949-2954.

65. Lee J-H, Terzaghi W, Gusmaroli G, Charron J-BF, Yoon H-J, Chen H, He YJ, Xiong $Y$, Deng XW: Characterization of Arabidopsis and rice DWD proteins and their roles as substrate receptors for CUL4-RING E3 ubiquitin ligases. Plant Cell 2008, 20:152-167.

66. Jin J, Arias EE, Chen J, Harper JW, Walter JC: A family of diverse Cul4Ddb1-interacting proteins includes $\mathrm{Cdt} 2$, which is required for $\mathrm{S}$ phase destruction of the replication factor Cdt1. Mol Cell 2006, 23:709-721.

67. Zhang $Y$, Feng $S$, Chen F, Chen H, Wang J, McCall C, Xiong Y, Deng XW Arabidopsis DDB1-CUL4 ASSOCIATED FACTOR1 forms a nuclear E3 ubiquitin ligase with DDB1 and CUL4 that is involved in multiple plant developmental processes. Plant Cell 2008, 20:1437-1455.

68. Angers S, Li T, Yi X, MacCoss MJ, Moon RT, Zheng N: Molecular architecture and assembly of the DDB1-CUL4A ubiquitin ligase machinery. Nature 2006, 443:590-593.

69. Pazhouhandeh M, Molinier J, Berr A, Genschik P: MSI4/FVE interacts with CUL4-DDB1 and a PRC2-like complex to control epigenetic regulation of flowering time in Arabidopsis. Pro Natl Acad Sci USA 2011, 108:3430-3435.

70. Bjerkan KN, Jung-Roméo S, Jürgens G, Genschik P, Grini PE: Arabidopsis WD repeat domain55 interacts with DNA damaged binding protein1 and is required for apical patterning in the embryo. Plant Cell 2012, 24:1013-1033.

71. Weigel D, Meyerowitz EM: The ABCs of floral homeotic genes. Cell 1994, 78:203-209.

72. Liu Z, Meyerowitz EM: LEUNIG regulates AGAMOUS expression in Arabidopsis flowers. Development 1995, 121:975-991.

73. Franks RG, Wang C, Levin JZ, Liu Z: SEUSS, a member of a novel family of plant regulatory proteins, represses floral homeotic gene expression with LEUNIG. Development 2002, 129:253-263.

74. Ho JW, Bishop E, Karchenko PV, Negre N, White KP, Park P: ChIP-chip versus ChIP-seq: Lessons for experimental design and data analysis. BMC Genomics 2011, 12:134.

75. Samach A, Onouchi H, Gold SE, Ditta GS, Schwarz-Sommer Z, Yanofsky MF, Coupland G: Distinct roles of CONSTANS target genes in reproductive development of Arabidopsis. Science 2000, 288:1613-1616

76. Borner R, Kampmann G, Chandler J, Gleissner R, Wisman E, Apel K, Melzer S: A MADS domain gene involved in the transition to flowering in Arabidopsis. Plant J 2000, 24:591-599.

77. Searle I, He Y, Turck F, Vincent C, Fornara F, Kröber S, Amasino RA Coupland G: The transcription factor FLC confers a flowering response to vernalization by repressing meristem competence and systemic signaling in Arabidopsis. Genes Dev 2006, 20:898-912.

78. Sawa M, Kay SA: GIGANTEA directly activates flowering locus $T$ in Arabidopsis thaliana. Proc Natl Acad Sci USA 2011, 108:11698-11703.

79. Nakamichi N, Kita M, Niinuma K, Ito S, Yamashino T, Mizoguchi T, Mizuno T: Arabidopsis clock-associated pseudo-response regulators PRR9, PRR7 and PRR5 coordinately and positively regulate flowering time through the canonical CONSTANS-dependent photoperiodic pathway. Plant Cell Physiol 2007, 48:822-832.

80. Fowler S, Lee K, Onouchi H, Samach A, Richardson K, Coupland G, Putterill J: GIGANTEA: a circadian clock-controlled gene that regulates photoperiodic flowering in Arabidopsis and encodes a protein with 
several possible membrane-spanning domains. EMBO J 1999, 18:4679-4688

81. Adrian J, Farrona S, Reimer JJ, Albani MC, Coupland G, Turck F: cisRegulatory elements and chromatin state coordinately control temporal and spatial expression of FLOWERING LOCUS T in Arabidopsis. Plant Cell 2010, 22:1425-1440.

82. Farrona S, Thorpe FL, Adrian J, Dong X, Sarid-Krebs L, Goodrich J, Turck F: Tissue-specific expression of FLOWERING LOCUS T in Arabidopsis is maintained independently of polycomb group protein repression. Plant Cell 2011, 9:3204-3214.

83. Goodrich J, Puangsomlee P, Martin M, Long D, Meyerowitz EM, Coupland G: A Polycomb-group gene regulates homeotic gene expression in Arabidopsis. Nature 1997, 386:44-51.

84. Amasino RM, Michaels SD: The timing of flowering. Plant Physiol 2010, $154: 516-520$

85. Jiang D, Wang Y, Wang Y, He Y: Repression of FLOWERING LOCUS C and FLOWERING LOCUS T by the Arabidopsis Polycomb repressive complex 2 components. PLoS One 2008, 3:e3404.

86. Wu X, Dabi T, Weigel D: Requirement of homeobox gene STIMPY/WOX9 for Arabidopsis meristem growth and maintenance. Curr Biol 2005, 15:436.

87. Bernier GJ: My favourite flowering image: the role of cytokinin as a flowering signal. J Exp Bot 2011.

88. Chaudhury AM, Letham S, Craig S, Dennis ES: amp1: A mutant with high cytokinin levels and altered embryonic pattern, faster vegetative growth, constitutive photomorphogenesis and precocious flowering. Plant J 1993, 4:907-916.

89. Immink RG, Tonaco IA, de Folter S, Shchennikova A, van Dijk AD, BusscherLange J, Borst JW, Angenent GC: SEPALLATA3: the 'glue' for MADS box transcription factor complex formation. Genome Biol 2009, 10:R24.

90. Wagner D, Meyerowitz EM: SPLAYED, a novel SWI/SNF ATPase homolog, controls reproductive development in Arabidopsis. Curr Biol 2002, 12:85-94.

91. Lenhard M, Bohnert A, Jürgens $G$, Laux T: Termination of stem cell maintenance in Arabidopsis floral meristems by interactions between WUSCHEL and AGAMOUS. Cell 2001, 105:805-814.

92. Mandel MA, Yanofsky MF: The Arabidopsis AGL9 MADS-box gene is expressed in young flower primordia. Sex Plant Reprod 1998, 11:22-28.

93. Kerstetter RA, Bollman K, Taylor RA, Bomblies K, Poethig RS: KANADI regulates organ polarity in Arabidopsis. Nature 2001, 411:706-709.

94. McConnell JR, Emery J, Eshed Y, Bao N, Bowman J, Barton MK: Role of PHABULOSA and PHAVOLUTA in determining radial patterning in shoots. Nature 2001, 411:709-713.

95. Sessions A, Nemhauser JL, McColl A, Roe JL, Feldmann KA, Zambryski PC: ETTIN patterns the Arabidopsis floral meristem and reproductive organs. Development 1997, 124:4481-4491.

96. Li H, He Z, Lu G, Lee SC, Alonso J, Ecker JR, Luan S: A WD40 domain cyclophilin interacts with histone $\mathrm{H} 3$ and functions in gene repression and organogenesis in Arabidopsis. Plant Cell 2007, 19:2403-2416.

97. Zeng L, Zhou M-M: Bromodomain: an acetyl-lysine binding domain. FEBS Letters 2002, 513:124-128.

98. Cunha PM, Sandmann T, Gustafson EH, Ciglar L, Eichenlaub MP, Furlong EE: Combinatorial binding leads to diverse regulatory responses: $\mathrm{Lmd}$ is a tissue-specific modulator of Mef2 activity. PloS Genet 2010, 6:e1001014.

99. Alonso JM, Stepanova AN, Leisse TJ, Kim CJ, Chen H, Shinn P, Stevenson DK, Zimmerman J, Barajas P, Cheuk R, Gadrinab C, Heller C, Jeske A, Koesema E, Meyers CC, Parker H, Prednis L, Ansari Y, Choy N, Deen H, Geralt M, Hazari N, Hom E, Karnes M, Mulholland C, Ndubaku R, Schmidt I, Guzman P, Aguilar-Henonin L, Schmid M, et al: Genome-Wide Insertional Mutagenesis of Arabidopsis thaliana. Science 2003, 301:653-657.

100. Murashige T, Skoog F: A rewised medium for rapid growth and bioassayswith tobacco tissue cultures. Physiologia Plantarum 1962, 15:473-497.

101. [http://www.clontech.com/].

102. Jiang $H$, Wong WH: SeqMap: mapping massive amount of oligonucleotides to the genome. Bioinformatics 2008, 24:2395-2396.

103. Ji H, Jiang H, Ma W, Johnson DS, Myers RM, Wong WH: An integrated software system for analyzing ChIP-chip and ChIP-seq data. Nat Biotechnol 2008, 11:1293-1300.
104. Naouar N, Vandepoele K, Lammens T, Casneuf T, Zeller G, van Hummelen P, Weigel D, Rätsch G, Inzé D, Kuiper M, De Veylder L, Vuylsteke M: Quantitative RNA expression analysis with Affymetrix Tiling 1.0R arrays identifies new E2F target genes. Plant I 2009, 57:184-194.

105. Maere S, Heymans K, Kuiper M: BiNGO: a Cytoscape plugin to assess overrepresentation of gene ontology categories in biological networks. Bioinformatics 2005, 21:3448-3449.

106. Cline MS, Smoot M, Cerami E, Kuchinsky A, Landys N, Workman C, Christmas R, Avila-Campilo I, Creech M, Gross B, Hanspers K, Isserlin R, Kelley R, Killcoyne S, Lotia S, Maere S, Morris J, Ono K, Pavlovic V, Pico AR, Vailaya A, Wang PL, Adler A, Hood L, Kuiper M, Sander C, Schmulevich I, Schwikowski B, Warner GJ, Ideker T, Bader GD: Integration of biological networks and gene expression data using Cytoscape. Nat Protoc 2007, 2:2366-2382.

107. [http://www.arabidopsis.org/]

108. [http://www.ambion.com/].

109. [http://www.promega.com]

110. [http://www.bio-rad.com].

111. Brambilla V, Battaglia R, Colombo M, Masiero S, Bencivenga S, Kater MM, Colombo L: Genetic and molecular interactions between BELL1 and MADS box factors support ovule development in Arabidopsis. Plant Cell 2007, 19:2544-2556.

112. [http://www.zeiss.com/].

113. Karimi M, Inzé D, Depicker A: GATEWAY vectors for Agrobacteriummediated plant transformation. Trends Plant Sci 2002, 7:193-195.

\section{doi:10.1186/gb-2013-14-6-r56}

Cite this article as: Gregis et al:: Identification of pathways directly regulated by SHORT VEGETATIVE PHASE during vegetative and reproductive development in Arabidopsis. Genome Biology 2013 14:R56.

\section{Submit your next manuscript to BioMed Central and take full advantage of:}

- Convenient online submission

- Thorough peer review

- No space constraints or color figure charges

- Immediate publication on acceptance

- Inclusion in PubMed, CAS, Scopus and Google Scholar

- Research which is freely available for redistribution 